AperTO - Archivio Istituzionale Open Access dell'Università di Torino

Moments of accumulated reward and completion time in Markovian models with application to unreliable manufacturing systems

This is a pre print version of the following article:

Original Citation:

Availability:

This version is available http://hdl.handle.net/2318/157008

since

Published version:

DOI:10.1016/j.peva.2014.02.005

Terms of use:

Open Access

Anyone can freely access the full text of works made available as "Open Access". Works made available under a Creative Commons license can be used according to the terms and conditions of said license. Use of all other works requires consent of the right holder (author or publisher) if not exempted from copyright protection by the applicable law. 


\title{
Moments of Accumulated Reward and Completion Time in Markovian Models with Application to Unreliable Manufacturing Systems
}

\author{
A. Angius ${ }^{\mathrm{a}}$, A. Horváth ${ }^{\mathrm{a}}$, M. Colledani ${ }^{\mathrm{b}}$ \\ ${ }^{a}$ Department of Computer Science, Universitàdi Torino, Turin, Italy, (e-mail: angius@di.unito.it and horvath@di.unito.it). \\ ${ }^{b}$ Mechanical Engineering Department, Politecnico di Milano, Milan, Italy, (e-mail: marcello.colledani@polimi.it).
}

\begin{abstract}
Performance evaluation models are used by companies to design, adapt, manage and control their production systems. In the literature, most of the effort has been dedicated to the development of efficient methodologies to estimate the first moment performance measures of production systems, such as the expected production rate, the buffer levels and the mean completion time. However, there is industrial evidence that the higher moments of the production output may drastically impact on the capability of managing the system operations, causing the observed system performance to be highly different from what expected. This paper presents a methodology to analyze the cumulated output and the lot completion time moments of Markovian reward models. Both the discrete and the continuous time cases are considered. The technique is applied to unreliable manufacturing systems characterized by general Markovian structures. Numerical results show how the theory developed in this paper can be applied to analyse the dependency of the output variability and the service level on the system parameters. Moreover, they highlight previously uninvestigated features of the system behavior that are useful while operating the system in practical settings.
\end{abstract}

Keywords: Performance Evaluation, Manufacturing Systems, Output Variability, General Markovian Machines, Markov Reward Models.

\section{Introduction}

\subsection{Motivation and Objective}

Manufacturing System Engineering methods have been developed in the last decades for investigating the dynamic behaviour of manufacturing systems, for estimating their performance and for supporting their efficient design, improvement and reconfiguration. Among these, simulation and analytical tools are the most commonly adopted methods. Typically, these approaches are focused on the first order performance measures of manufacturing systems, such as the average throughput, the average work in progress and the average system flow time. During the system design phase, these tools are used to select system solutions that profitably exploit the trade-offs between these first order performance measures. The higher moments of the system performance measures are generally difficult to analyze and are rarely considered.

However, in the presence of random events and disturbances in the production, higher moments of the performance measures are relevant to correctly predict the system output. For example, due to the production variability, the observed quantity of produced items on a limited time horizon can be highly different from its expected value. This variability mines the ability to meet customer orders on time and to ensure the required service level of the system. This problem may directly corrupt the profitability of the system. Indeed, systems designed only by considering their mean performance levels show poor robustness to disturbances. Low output variance indicates stability of the output of the production system, less unforeseen delays and small fraction of escaped orders, which translates to lower production costs, ultimately leading to improved company competitiveness. On the contrary, high variance means instability of the output and significant fluctuations in production quantities observed on a day by day basis.

Typical sources of variability in production systems are random failure occurrences and durations. A real case in the automotive industry ([6]) reports that for a system composed of 22 machines affected by the 
occurrences of 144 different failure modes the weekly cumulated production has a coefficient of variation, estimated from the available field data over three months, equal to 0.263 . Thus, it is highly probable that the weekly demand will not be met since the system was designed only considering its long term expected performance.

This problem has recently become even more critical in the manufacturing context, since frequent external demand fluctuations and unpredictable shortages are more and more commonly experienced. For example, since 2008 the German automotive market has experienced quarterly demand fluctuations varying between 300000 units and 600000 units. In this situation, the combined effect of internal production fluctuations and external demand variability can directly translate into overall insufficient company's performance. Therefore, developing tools to understand, analyze, and control the higher moments of the production system output is of increasing paramount importance for the manufacturing industry.

\subsection{Literature Review}

In spite of the relevance of this issue in industry, the number of papers discussing the higher moments of the output in production lines is still relatively limited and the underlying assumptions of the available methods are simplistic, thus preventing their wide application in industry. Existing research contributions on the analysis of higher moments of manufacturing system performance measures are solely devoted to the analysis of the production variance, considering both the cumulated production and the interdeparture times of the output process.

The output variability of production lines was originally studied in [22]. The author proposed an exact numerical method to calculate the first two moments of the asymptotic output measures, i.e. the asymptotic throughput and variance rate, that is the limiting variance of the output per time unit. The method considered small buffered production lines featuring unreliable machines with geometric/exponential failure and repair times. His approach is based on the state-space representation of the system and the use of the inverse of the fundamental matrix, thus only simple systems with small number of machines and buffer capacities can be analyzed with success.

[13] presented an approach to estimate the asymptotic variance rate of interdeparture times in production lines with exponential processing times, perfectly reliable machines, and finite buffer capacities. This work was later extended in [14] to model machines with general processing times. In this paper, it was observed by simulation that by increasing the skewness of the processing times the inter-departure variance also increases. The complexity of Hendrick's approach is comparable to [22], being dependent on the number of states representing the system.

[26] obtained a closed form expression of the variance of the lead time in a two machine line in which machines may fail in only one mode. Moreover, a formula for the calculation of the variance of the output of a single machine with a single failure mode was derived [10]. The method is based on the solution of the difference equation describing the system dynamics. [2] extended the method of Gershwin to the case of the isolated machine with multiple failure modes. The developed method was then used to approximate the performance of production lines through a decomposition approach. The effect of previous stages on the last machine in the system is considered by adjusting the failure and repair parameters of the single machine model. However, the method is shown to have large errors in the variance estimation (around 20\% compared to simulation results) since the adopted decomposition equations [11] did not capture and propagate the output variability throughout the line.

Tan proposed a series of studies on the output variability of production systems. These works include the analysis and calculation of the output variability for machines in isolation, multi-stage unbuffered lines, production lines with parallel and series machines and small buffered manufacturing systems. He proposed both continuous time models [27, 29, 28] and discrete time models [30, 31, 32] for the analysis. In terms of investigated machine models, the studies include reliable machines with exponential processing times [27], unreliable machines with a single failure mode featuring geometrically or exponentially distributed failure times [31] and unreliable machines with Coxian distributed repair time [29]. The asymptotic variance rate and the approximate service level of the system are considered as performance measures of interest. In terms of methods, Tan proposed a matrix-geometric approach for the estimation of the asymptotic variance rate in 
buffered systems. Compared to Miltenburg's approach, the method proposed by Tan is much more efficient in terms of number of executed floating point operations. The method uses the Grassman approach [12] to iteratively obtain the performance of interest. The complexity of the adopted procedure depends both on the length of the observed time period and on the size of the Markov chain describing the process, thus on the buffer capacity.

In [5] a fluid Markovian model was proposed to derive an exact closed-form formula to calculate the first two moments of the asymptotic output for unreliable machines with generally distributed up and down times. An attempt to extend this approach to two-machine one buffer dipoles was made, by approximating the dipole behavior with an equivalent single machine having two switching operational modes. When the second machine is not starved, the equivalent machine is exactly identical to the second machine of the dipole; when the second machine is starved, the equivalent machine behaves as the first machine in the dipole. However, the autocorrelation structure of the starvation times was not considered, thus this approximation is shown to perform poorly in specific configurations.

In [18] the variance of the output for production lines composed of unreliable machines and finite buffers is derived. The most limiting assumption to the application of the proposed method is the Bernoulli reliability model, which assumes repair time equal to the cycle time of the machines. The authors focused on the "due time performance" which is an equivalent measure of the service level. Recently, a similar approach was proposed to study the transient behavior of production systems ([19] and [21]).

The transient behavior of production systems was also addressed in [9] and [4]. In fact,the work of [9] studied both the transient and steady-state variability in the output of small buffered lines with reliable machines featuring exponentially distributed processing times, by adopting n-fold convolution of the interarrival and the processing time distributions. [4] focused on the system output mean and variance during the transient period. The approach models long unbuffered production lines with unreliable machines subject to a single failure mode, with exponentially distributed failure and repair times, by using a sample path method.

Recently, an approximate method was proposed by [20] to study long assembly lines with finite buffers and general service times. Their approach used a two-moment approximation to estimate the output variability in the assembly line, by measuring the coefficient of variation of inter-departure time.

The effect of the autocorrelation structure proposed by [13] have been further investigated by [7], for small systems featuring unreliable machines affected by multiple failure modes. The authors extended this approach to approximately evaluate the asymptotic variance rate of multi-stage production lines with machines having multiple geometric failure modes [8]. The proposed decomposition method suffers the same limitations of the decomposition method proposed in [10].

\subsection{Contribution}

As reported in the previous section, the available methods that are applicable to multi-stage systems only consider simple serial system layouts under the assumptions of exponentially or, in the discrete time domain, geometrically distributed machine failure and repair times. However, real systems commonly present more complex structures and material flows. Moreover, while in real systems the times to machine failures can often be modeled using exponential or geometrical distributions with acceptable accuracy, given the mechanical and electronic nature of failures, the times to repair are rarely observed to follow exponential distributions ([16]).

This paper considers general Markovian machines and systems in the analysis, thus enabling to revise these critical assumptions of the existing methods. In these more realistic settings, very little is known on how to manage production systems to smooth and control the variability of their output. Moreover, in terms of considered performance measures, only the cumulated production and the inter-departure times have attracted interest in the past. However, production managers are generally interested also in time-related performance measures, such as the system completion time, or in quantiles of distributions of performance measures, to answer important questions like "What is the due date to be quoted for a given order?" and "What is the probability of delivering a given order on time, under a particular system configuration?". In addition, only the second moment of the production output has been considered in previous works. However, 
in order to better approximate the system service level of the system, higher moments should be considered, especially in the short-medium run.

To overcome these limitations, the objective of this paper is to develop a general theory and a methodology to analyze the moments of the cumulated output and the moments of the completion time in manufacturing systems modeled as arbitrarily complex Markov chains. Grounding on the exact calculation of the moments of the distribution of these performance measures, the quantiles of the distributions can also be approximated. The generality of the proposed approach allows modeling and studying the moments of the output variability under many different system configurations within a unique framework, also including several previously uninvestigated system layouts. Among these, unbuffered multi-stage serial-parallel systems with limited repair capacity, systems with machines producing in batches, and buffered two-machine lines with degrading machines are modeled and analyzed as examples in this paper, within the proposed framework. Moreover, the impact of the main system and machine parameters on the variability of the output is investigated, with the objective of deriving new system design rules for reducing the variability and meeting the due-time performance of the system.

\subsection{Paper Organization}

The paper is organized as follows. In Section 2 we discuss Markov reward models reporting existing results and deriving new ones. In Section 3 we illustrate the application of the proposed framework on several examples. Conclusions are drawn in Section 4.

\section{Markov Reward Models}

In a Markov reward model (MRM) an underlying Markov chain modulates the reward rate. A sojourn of length $u$ in an up-state accumulates a quantity of reward proportional to $u$ while in a down-state the production is zero. The generality of modeling machines with MRMs lies in the facts that

- MRMs allow us to model general failure/repair mechanisms with phase type distributed durations;

- the set of phase type distributions is dense in the field of all positive-valued distributions ([25]), i.e., it can be used to approximate any failure/repair distribution;

- there exists methods to construct phase type distributions (either continuous or discrete) with given moments (see, e.g., [1, 35]).

While to compute the distribution of the accumulated reward and the distribution of completion time is computationally heavy, there exist efficient methods for the calculation of the moments of these distributions. In Section 2.1 we report these results for continuous time models with continuous reward and in Section 2.2 we derive the counterpart for discrete time models with discrete reward. The corresponding quantities will be denoted by the same symbols in the continuous and the discrete case.

\subsection{Continuous Markov Reward Model}

A continuous time Markov reward model is defined by the infinitesimal generator of the underlying continuous time Markov chain (CTMC), denoted by $\mathbf{Q}$, and the reward rates associated with the states denoted by $r_{i}$ for state $i$ and organized into a diagonal matrix denoted by $\mathbf{R}$. During a sojourn of length $u$ in state $i$ the system accumulates $u r_{i}$ amount of reward. As a simple example consider a model composed of two states described by the following infinitesimal generator and rate matrix:

$$
\mathbf{Q}=\left|\begin{array}{cc}
-1.5 & 1.5 \\
2.5 & -2.5
\end{array}\right|, \quad \mathbf{R}=\left|\begin{array}{cc}
1.2 & 0 \\
0 & 2.2
\end{array}\right|
$$

which implies that reward is gained in the first (second) state of the CTMC at rate 1.2 (2.2). The graphical representation and a possible trajectory for both the underlying CTMC and the accumulated reward are depicted in Figure 1. 

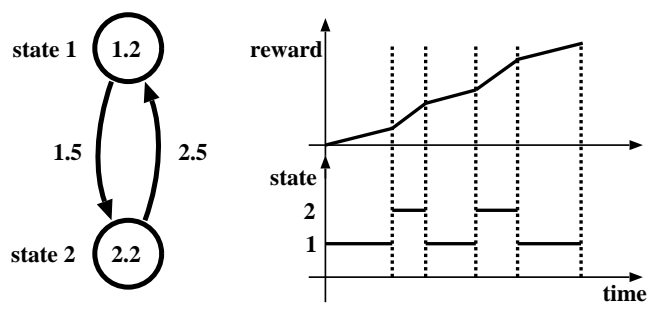

Figure 1: Example continuous Markov reward model: Markov chain (left) and a possible trajectory (right)

Denoting by $X(t)$ the state of the chain at time $t$ and by $Z(t)$ the accumulated reward (the production), we have $Z(t)=\int_{s=0}^{t} r_{X(s)} d s$. The completion time, i.e. the amount of time necessary to produce a given quantity $x$, is denoted by $C(x)$ and defined as $C(x)=\min [t \geq 0: Z(t)=x]$. The quantities characterizing the accumulated reward and the completion time are

$$
\begin{aligned}
& F_{i j}(t, x)=P\{X(t)=j, Z(t)<x \mid X(0)=i\} \\
& G_{i j}(t, x)=P\{X(C(x))=j, C(x)<t \mid X(0)=i\}
\end{aligned}
$$

where

- $F_{i j}(t, x)$ is the joint distribution of the accumulated reward and the state of the underlying CTMC supposing that the initial state is $i$ and

- $G_{i j}(t, x)$ is the joint probability of the completion time and the state at completion supposing that the initial state is $i$.

The matrices formed of the above quantities, $\mathbf{F}(t, x)=\left[F_{i j}(t, x)\right]$ and $\mathbf{G}(t, x)=\left[G_{i j}(t, x)\right]$, can be described in double transform domain which leads to the following expressions $([17,34])$

$$
\begin{gathered}
\mathbf{F}^{* *}(s, v)=\frac{1}{v}(s \mathbf{I}+v \mathbf{R}-\mathbf{Q})^{-1} \\
\mathbf{G}^{* *}(s, v)=\frac{1}{s}(s \mathbf{I}+v \mathbf{R}-\mathbf{Q})^{-1} \mathbf{R}
\end{gathered}
$$

where $f^{*}(s)=\int_{t=0}^{\infty} f(t) e^{-s t} d t$ denotes the Laplace transform which is applied both according to time $(t \rightarrow s)$ and accumulated reward $(x \rightarrow v)$ and $\mathbf{I}$ denotes the identity matrix. In theory, both the accumulated reward and the completion time can be analyzed based on (3) and (4) by numerical or symbolic inverse Laplace transformation. In practice, this approach works only for small and/or specially structured MRMs.

For larger MRMs, as it was proposed in [37], the moments of the quantity defined in (1) can be computed efficiently. To this purpose define the moments of the state dependent accumulated reward as

$$
K_{i j}^{(n)}(t)=\int_{x=0}^{\infty} x^{n} d F_{i j}(t, x), n=0,1,2, \ldots
$$

such that $K_{i j}^{(n)}(t)$ is the $n$th moment of the accumulated reward after $t$ time units multiplied by the probability that the state at time $t$ is $j$ supposing that the initial state is $i$. Note that if $n=0$ then (5) corresponds simply to the transient probabilities of the underlying CTMC. With the definition in (5), the mean of the accumulated reward after $t$ time units given that the initial state is $i$ is given by $\sum_{j} K_{i j}^{(1)}(t)$. It was shown in [36] that the matrices $\mathbf{K}^{(n)}(t)=\left[K_{i j}^{(n)}(t)\right], n=0,1,2, \ldots$, satisfy the differential equations

$$
\frac{d \mathbf{K}^{(n)}(t)}{d t}=n \mathbf{K}^{(n-1)}(t) \mathbf{R}+\mathbf{K}^{(n)}(t) \mathbf{Q} .
$$



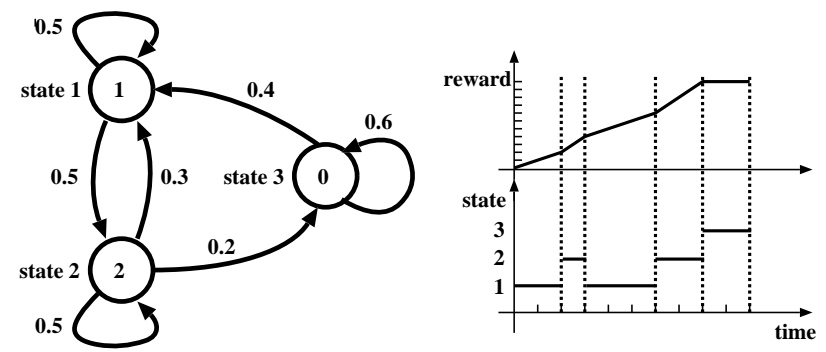

Figure 2: Example discrete Markov reward model: Markov chain (left) and a possible trajectory (right)

In [37] the authors proposed efficient randomization based methods for the calculation of $\mathbf{K}^{(n)}(t)$ for $n=$ $1, \ldots, N$ whose time complexity is roughly $\sum_{i=0}^{N}(i+1)$ times higher than the complexity of the transient analysis of the CTMC underlying the MRM. The space complexity is instead $N+1$ higher than for the transient analysis of the CTMC. The same authors propose a method for the calculation of the moments of the completion time as well but this method is efficient only if the number of states with zero reward rate is low.

\subsection{Discrete Markov Reward Model}

Discrete Markov Reward Models are relevant in the field of manufacturing system modeling. Indeed, in discrete part manufacturing, the processing time of machines is usually deterministic. Moreover, in many cases, the machines are synchronous, in the sense that they start and finish the production simultaneously, at fixed points in time. These features are more realistically captured within the framework of discrete time models.

In the discrete case a discrete time Markov chain (DTMC) modulates the accumulation of reward and a discrete amount of reward is gained in each time slot (see, e.g., [23]). Let $\mathbf{P}$ denote the transition probability matrix of the underlying DTMC and $r_{i}, 0 \leq r_{i} \leq c$, the integer reward rate associated with state $i$ where $c$ is the maximum reward per step. A sojourn of length $l, l=1,2,3, \ldots$, in state $i$ provides $l r_{i}$ reward. As a simple example consider a model composed of three states described by the following transition matrix and reward rates

$$
\mathbf{P}=\left|\begin{array}{ccc}
0.5 & 0.5 & 0 \\
0.3 & 0.5 & 0.2 \\
0.4 & 0 & 0.6
\end{array}\right|, r_{1}=1, r_{2}=2, r_{3}=0 .
$$

The graphical representation and a possible trajectory for both the underlying DTMC and the accumulated reward are depicted in Figure 2.

Denoting by $X_{n}$ the state of the chain after the $n$th transition and by $Z_{n}$ the reward accumulated in the first $n$ steps, we have $Z_{n}=\sum_{i=0}^{n-1} r_{X_{i}}$ for $n=1,2,3, \ldots$. Further, let $S_{i}, 0 \leq i \leq c$, denote the set of states with reward rate $i$ and define the matrices $\mathbf{P}^{(i)}, 0 \leq i \leq c$, with entries according to

$$
\mathbf{P}_{k l}^{(i)}= \begin{cases}\mathbf{P}_{k l} & \text { if } k \in S_{i} \\ 0 & \text { otherwise. }\end{cases}
$$

For the example model introduced above we have

$$
\mathbf{P}^{(0)}=\left|\begin{array}{ccc}
0 & 0 & 0 \\
0 & 0 & 0 \\
0.4 & 0 & 0.6
\end{array}\right|, \quad \mathbf{P}^{(1)}=\left|\begin{array}{ccc}
0.5 & 0.5 & 0 \\
0 & 0 & 0 \\
0 & 0 & 0
\end{array}\right|, \quad \mathbf{P}^{(2)}=\left|\begin{array}{ccc}
0 & 0 & 0 \\
0.3 & 0.5 & 0.2 \\
0 & 0 & 0
\end{array}\right|
$$

Our aim is to characterise the joint probability of the accumulated reward and the background state defined for $n=0,1,2, \ldots$ and $k=0,1,2, \ldots$ as

$$
F_{i j}(n, k)=\operatorname{Pr}\left\{Z_{n}=k, X_{n}=j \mid X_{0}=i\right\} .
$$


It is easy to see that the following recursion holds for $n=1,2,3, \ldots$ and $k=0,1,2, \ldots$

$$
F_{i j}(n, k)=\sum_{m=0}^{\min (k, c)} \sum_{l \in S_{m}} F_{i l}(n-1, k-m) \mathbf{P}_{l j}
$$

which, introducing the matrix notation $\mathbf{F}(n, k)=\left[F_{i j}(n, k)\right]$, becomes

$$
\mathbf{F}(n, k)=\sum_{m=0}^{\min (k, c)} \mathbf{F}(n-1, k-m) \mathbf{P}^{(m)}
$$

The following theorem provides the discrete counterpart of (3).

Theorem 1. The double z-transform of $\mathbf{F}(n, k)$ is given by

$$
\mathbf{F}^{* *}\left(z_{1}, z_{2}\right)=\left(\mathbf{I}-z_{1} \sum_{m=0}^{c} z_{2}^{m} \mathbf{P}^{(m)}\right)^{-1}
$$

Proof. Multiplying the left hand side of (7) by $z_{1}^{n} z_{2}^{k}$ and summing for $n=1,2, \ldots$ and $k=0,1, \ldots$ gives

$$
\begin{gathered}
\sum_{n=1}^{\infty} \sum_{k=0}^{\infty} z_{1}^{n} z_{2}^{k} \mathbf{F}(n, k)=\mathbf{F}^{* *}\left(z_{1}, z_{2}\right)-\sum_{k=0}^{\infty} z_{2}^{k} \mathbf{F}(0, k)= \\
\mathbf{F}^{* *}\left(z_{1}, z_{2}\right)-\mathbf{I}
\end{gathered}
$$

because $\mathbf{F}(0,0)=\mathbf{I}$ and $\mathbf{F}(0, k)=\mathbf{0}$ for $k=1,2,3, \ldots$. By performing the same operation on the right hand side of $(7)$ we have

$$
\begin{aligned}
& \sum_{n=1}^{\infty} \sum_{k=0}^{\infty} z_{1}^{n} z_{2}^{k} \sum_{m=0}^{\min (k, c)} \mathbf{F}(n-1, k-m) \mathbf{P}^{(m)}= \\
& \sum_{m=0}^{c} \sum_{n=1}^{\infty} \sum_{k=m}^{\infty} z_{1}^{n} z_{2}^{k} \mathbf{F}(n-1, k-m) \mathbf{P}^{(m)}= \\
& z_{1} \sum_{m=0}^{c} z_{2}^{m} \sum_{n=1}^{\infty} \sum_{k=m}^{\infty} z_{1}^{n-1} z_{2}^{k-m} \mathbf{F}(n-1, k-m) \mathbf{P}^{(m)}= \\
& z_{1} \sum_{m=0}^{c} z_{2}^{m} \sum_{n=0}^{\infty} \sum_{k=0}^{\infty} z_{1}^{n} z_{2}^{k} \mathbf{F}(n, k) \mathbf{P}^{(m)}= \\
& z_{1} \sum_{m=0}^{c} z_{2}^{m} \mathbf{F}^{* *}\left(z_{1}, z_{2}\right) \mathbf{P}^{(m)}
\end{aligned}
$$

From (9) and (10) we have

$$
\mathbf{F}^{* *}\left(z_{1}, z_{2}\right)-\mathbf{I}=z_{1} \sum_{m=0}^{c} z_{2}^{m} \mathbf{F}^{* *}\left(z_{1}, z_{2}\right) \mathbf{P}^{(m)}
$$

which by simple rearrangements provides the theorem.

The joint distribution of the completion time and the state is defined for $n=1,2,3, \ldots$ and $k=1,2,3, \ldots$ as

$$
G_{i j}(n, k)=\operatorname{Pr}\left\{Z_{n} \geq k, Z_{n-1}<k, X_{n}=j \mid X_{0}=i\right\} .
$$

It is easy to see that the following relation holds for $n=1,2,3, \ldots$ and $k=1,2,3, \ldots$

$$
G_{i j}(n, k)=\sum_{m=1}^{\min (k, c)} \sum_{h=m}^{c} \sum_{l \in S_{h}} F_{i l}(n-1, k-m) \mathbf{P}_{l j}
$$


which, by the matrix notation $\mathbf{G}(n, k)=\left[G_{i j}(n, k)\right]$, becomes

$$
\mathbf{G}(n, k)=\sum_{m=1}^{\min (k, c)} \sum_{h=m}^{c} \mathbf{F}(n-1, k-m) \mathbf{P}^{(h)} .
$$

The following theorem provides the discrete counterpart of (4).

Theorem 2. The double $z$-transform of $\mathbf{G}(n, k)$ is

$$
\begin{aligned}
& \mathbf{G}^{* *}\left(z_{1}, z_{2}\right)= \\
& \mathbf{I}+\sum_{m=1}^{c} \sum_{h=m}^{c} z_{1} z_{2}^{m}\left(\mathbf{I}-z_{1} \sum_{m=0}^{c} z_{2}^{m} \mathbf{P}^{(m)}\right)^{-1} \mathbf{P}^{(h)}
\end{aligned}
$$

Proof. Multiplying the left hand side of (11) by $z_{1}^{n} z_{2}^{k}$ and summing for $n=1,2, \ldots$ and $k=0,1, \ldots$ gives

$$
\begin{aligned}
\sum_{n=1}^{\infty} \sum_{k=0}^{\infty} z_{1}^{n} z_{2}^{k} \mathbf{G}(n, k)= & \mathbf{G}^{* *}\left(z_{1}, z_{2}\right)-\sum_{k=0}^{\infty} \mathbf{G}(0, k)= \\
& \mathbf{G}^{* *}\left(z_{1}, z_{2}\right)-\mathbf{I}
\end{aligned}
$$

because $\mathbf{G}(0,0)=\mathbf{I}$ and $\mathbf{G}(0, k)=\mathbf{0}$ for $k=1,2,3, \ldots$ By performing the same operation on the right hand side of (11) we have

$$
\begin{aligned}
& \sum_{n=1}^{\infty} \sum_{k=0}^{\infty} z_{1}^{n} z_{2}^{k} \sum_{m=1}^{\min (k, c)} \sum_{h=m}^{c} \mathbf{F}(n-1, k-m) \mathbf{P}^{(h)}= \\
& \sum_{m=1}^{c} \sum_{h=m}^{c} z_{1} z_{2}^{m} \sum_{n=1}^{\infty} \sum_{k=m}^{\infty} z_{1}^{n-1} z_{2}^{k-m} \mathbf{F}(n-1, k-m) \mathbf{P}^{(h)}= \\
& \sum_{m=1}^{c} \sum_{h=m}^{c} z_{1} z_{2}^{m} \mathbf{F}^{* *}\left(z_{1}, z_{2}\right) \mathbf{P}^{(h)}
\end{aligned}
$$

from which by applying (8) the theorem follows.

\subsubsection{Moments of accumulated reward}

As in the continuous case, in theory, the accumulated reward and the completion time can be analysed based on (8) and (12) by applying double inverse z-transform. This approach is viable for small or highly structured DTMCs. The following theorem, which is the discrete counterpart of (6), provides an efficient approach for the calculation of the moments of the accumulated reward defined as

$$
\mathbf{K}^{(l)}(n)=\sum_{k=0}^{\infty} k^{l} \mathbf{F}(n, k), k=0,1,2, \ldots
$$

Theorem 3. The moments of the accumulated reward, $\mathbf{K}^{(l)}(n), k=0,1,2, \ldots$, satisfy the following recursive relation

$$
\mathbf{K}^{(l)}(n)=\sum_{i=0}^{l}\left(\begin{array}{l}
l \\
i
\end{array}\right) \mathbf{K}^{(l-i)}(n-1) \sum_{m=0}^{c} m^{i} \mathbf{P}^{(m)} .
$$

Proof. The moments $\mathbf{K}^{(l)}(n)$ can be derived starting from (7) by multiplying both sides by $k^{l}$ and summing up for $k=0,1,2, \ldots$ which leads to

$$
\mathbf{K}^{(l)}(n)=\sum_{k=0}^{\infty} k^{l} \sum_{m=0}^{\min (k, c)} \mathbf{F}(n-1, k-m) \mathbf{P}^{(m)}
$$


from which by changing the order of the summation and applying

$$
k^{l}=\sum_{i=0}^{l}\left(\begin{array}{l}
l \\
i
\end{array}\right)(k-m)^{l-i} m^{i}
$$

we get

$$
\begin{aligned}
\mathbf{K}^{(l)}(n)= & \sum_{m=0}^{c} \sum_{k=m}^{\infty} \sum_{i=0}^{l}\left(\begin{array}{l}
l \\
i
\end{array}\right)(k-m)^{l-i} m^{i} \mathbf{F}(n-1, k-m) \mathbf{P}^{(m)}= \\
& \sum_{m=0}^{c} \sum_{i=0}^{l}\left(\begin{array}{l}
l \\
i
\end{array}\right) \sum_{k=m}^{\infty}(k-m)^{l-i} \mathbf{F}(n-1, k-m) m^{i} \mathbf{P}^{(m)}= \\
& \sum_{m=0}^{c} \sum_{i=0}^{l}\left(\begin{array}{l}
l \\
i
\end{array}\right) \mathbf{K}^{(l-i)}(n-1) m^{i} \mathbf{P}^{(m)}
\end{aligned}
$$

which yields the expression given in (13).

The base cases of the recursion are given simply by the matrices

$$
\mathbf{K}^{(l)}(0)= \begin{cases}\mathbf{I} & \text { if } l=0 \\ \mathbf{Z} & \text { otherwise }\end{cases}
$$

where $\mathbf{Z}$ denotes a matrix of zeros.

The recursion given in (7) for the distribution of the accumulated reward is based on the so-called forward scheme. It is easy to verify that the following backward counterpart also holds

$$
\mathbf{F}(n, k)=\sum_{m=0}^{\min (k, c)} \mathbf{P}^{(m)} \mathbf{F}(n-1, k-m)
$$

and it leads to the following theorem which is the backward counterpart of Theorem 3 and thus provides a backward scheme for the computation of the moments of the accumulated reward.

Theorem 4. The moments of the accumulated reward, $\mathbf{K}^{(l)}(n), k=0,1,2, \ldots$, satisfy the following backward recursive relation

$$
\mathbf{K}^{(l)}(n)=\sum_{i=0}^{l}\left(\begin{array}{l}
l \\
i
\end{array}\right) \sum_{m=0}^{c} m^{i} \mathbf{P}^{(m)} \mathbf{K}^{(l-i)}(n-1) .
$$

Proof. Follows the same path used for Theorem 3.

The difference between the forward scheme (Theorem 3) and the backward scheme (Theorem 4) is relevant when a vector based calculation of the moments of the accumulated reward is implemented. With the forward scheme an arbitrary initial distribution over all states can be considered and the computations provide the probability of each state at the end of the transient analysis period. With the backward scheme, with one transient analysis, all states as deterministic initial state can be analyzed but the identity of the final state is lost.

The expressions given in Theorems 3 and 4 directly provide a procedure for the calculation of the moments of the accumulated reward. The time complexity of calculating $\mathbf{K}^{(l)}(n)$ for $l=1, \ldots, N$ is roughly $\sum_{i=0}^{N}(i+1)$ times the complexity of the transient analysis of the underlying DTMC. The space complexity is instead $N+1$ higher than for the transient analysis of the DTMC.

In the following we explain the vector based use of the forward scheme to calculate the first two moments of the accumulated reward assuming an initial probability distribution of the states, given by the vector $\boldsymbol{\pi}(0)$. Let us use the notation

$$
\mathbf{f}^{(l)}(n)=\boldsymbol{\pi}(0) \mathbf{K}^{(l)}(n)
$$


with which $\mathbf{f}^{(0)}(n)$ is the vector of the state probabilities after $n$ steps and $\mathbf{f}^{(l)}(n), l \geq 1$ is the vector containing the information on the $l$ th moment of the accumulated reward after $n$ steps. By multiplying the right hand side of $(13)$ by $\boldsymbol{\pi}(0)$ from the left, we obtain a recursion for the vectors $\mathbf{f}^{(l)}(n)$. Writing this recursion explicitly for $l=0,1,2$ results in

$$
\begin{aligned}
& \mathbf{f}^{(0)}(n)=\mathbf{f}^{(0)}(n-1) \sum_{m=0}^{c} \mathbf{P}^{(m)}=\mathbf{f}^{(0)}(n-1) \mathbf{P} \\
& \mathbf{f}^{(1)}(n)=\mathbf{f}^{(1)}(n-1) \mathbf{P}+\mathbf{f}^{(0)}(n-1) \sum_{m=0}^{c} m \mathbf{P}^{(m)} \\
& \mathbf{f}^{(2)}(n)=\mathbf{f}^{(2)}(n-1) \mathbf{P}+2 \mathbf{f}^{(1)}(n-1) \sum_{m=0}^{c} m \mathbf{P}^{(m)}+\mathbf{f}^{(0)}(n-1) \sum_{m=0}^{c} m^{2} \mathbf{P}^{(m)}
\end{aligned}
$$

The mean accumulated reward can be obtained then by summing up all entries of $\mathbf{f}^{(1)}(n)$ while the second moment is the sum of the entries of $\mathbf{f}^{(2)}(n)$. The initial values for the recursion are obtained considering (14) and they are simply

$$
\mathbf{f}^{(0)}(0)=\boldsymbol{\pi}(0), \mathbf{f}^{(1)}(0)=|0, \ldots, 0|, \mathbf{f}^{(2)}(0)=|0, \ldots, 0|
$$

The vector based application of the backward scheme can be obtained by defining the column vectors

$$
\mathbf{b}^{(l)}(n)=\mathbf{K}^{(l)}(n) \mathbf{1}
$$

where $\mathbf{1}$ is a column vector with all entries equal to 1 . The $i$ th entry of $\mathbf{b}^{(l)}(n)$ gives the $l$ th moment of the accumulated reward given that the chain started in state $i$. By multiplying by $\mathbf{1}$ both sides of (16) from the right, we obtain a recursive scheme to calculate $\mathbf{b}^{(l)}(n)$. For the first two moments we have

$$
\begin{aligned}
& \mathbf{b}^{(0)}(n)=\sum_{m=0}^{c} \mathbf{P}^{(m)} \mathbf{b}^{(0)}(n-1)=\mathbf{P} \mathbf{b}^{(0)}(n-1) \\
& \mathbf{b}^{(1)}(n)=\mathbf{P} \mathbf{b}^{(1)}(n-1)+\sum_{m=0}^{c} m \mathbf{P}^{(m)} \mathbf{b}^{(0)}(n-1) \\
& \mathbf{b}^{(2)}(n)=\mathbf{P} \mathbf{b}^{(2)}(n-1)+2 \sum_{m=0}^{c} m \mathbf{P}^{(m)} \mathbf{b}^{(1)}(n-1)+\sum_{m=0}^{c} m^{2} \mathbf{P}^{(m)} \mathbf{b}^{(0)}(n-1)
\end{aligned}
$$

The initial values of the recursion, based on (14), are

$$
\mathbf{b}^{(0)}(0)=|1, \ldots, 1|^{T}, \mathbf{b}^{(1)}(0)=|0, \ldots, 0|^{T}, \mathbf{b}^{(2)}(0)=|0, \ldots, 0|^{T}
$$

\subsubsection{Moments of completion time}

In the following we derive an efficient, recursive scheme for the computation of the moments of the completion time. To this end, let us use the notation

$$
G_{i j}(n, k, e)=\operatorname{Pr}\left\{Z_{n}=k+e, Z_{n-1}<k, X_{n}=j \mid X_{0}=i\right\}
$$

which characterizes not only the time instance of reaching a given amount of reward but also the excess amount upon the moment of completion. In matrix notation we write $\mathbf{G}(n, k, e)=\left[G_{i j}(n, k, e)\right]$. The $m$ th moment of the completion time with a given amount of excess will be denoted by

$$
L_{i j}^{(m)}(k, e)=\sum_{n=0}^{\infty} n^{m} G_{i j}(n, k, e)
$$




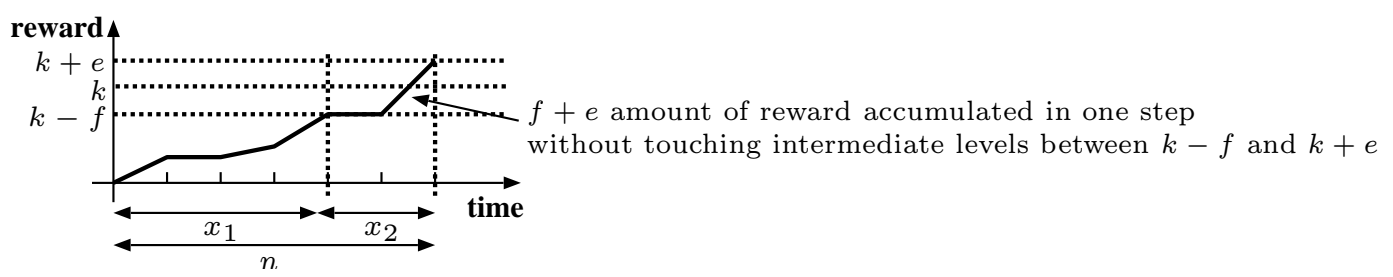

Figure 3: A possible way to reach $k$ amount of reward with $e$ amount of excess

with associated matrix notation $\mathbf{L}^{(m)}(k, e)=\left[L_{i j}^{(m)}(k, e)\right]$. It follows that, by summing up for all possible values of the amount of excess, the moments of the completion time, $\mathbf{L}^{(m)}(k)$, can be obtained as

$$
\mathbf{L}^{(m)}(k)=\sum_{e=0}^{c-1} \mathbf{L}^{(m)}(k, e)
$$

The following theorem provides a recursive relation for the quantities $\mathbf{L}^{(m)}(k, e)$.

Theorem 5. The moments of the completion time with a given amount of excess, $e, 0 \leq e \leq c-1$, satisfies the forward recursion

$$
\mathbf{L}^{(m)}(k, e)=\sum_{i=0}^{m} \sum_{f=1}^{c-e}\left(\begin{array}{c}
m \\
i
\end{array}\right) \mathbf{L}^{(i)}(k-f, 0) \mathbf{L}^{(m-i)}(1, f-1+e)
$$

Proof. The recursion is based on the equation

$$
\begin{aligned}
& L_{i j}^{(m)}(k, e)=\sum_{n=0}^{\infty} n^{m} G_{i j}(n, k, e)= \\
& \sum_{x_{1}=0,1, \ldots x_{2}=0,1, . .} \sum_{f=1}\left(x_{1}+x_{2}\right)^{m} \sum_{l \in S}^{c-e} \sum_{i l} G_{i l}\left(x_{1}, k-f, 0\right) G_{l j}\left(x_{2}, 1, f-1+e\right)
\end{aligned}
$$

where we exploited the fact that all possible ways to reach $k$ amount of reward with $e$ amount of excess is composed of two parts

- reaching a level $k-f$ with zero excess where $1 \leq f \leq c-e\left(\operatorname{described}\right.$ by $\left.G_{i l}\left(x_{1}, k-f, 0\right)\right)$, and then

- reaching level $k$ with excess $e$ (i.e., arriving to level $k+e$ ) without "touching" any level between $k-f$ and $k+e$ (described by $G_{l j}\left(x_{2}, 1, f-1+e\right)$ which guarantees that the accumulated reward has not reached level $k$ before $x_{1}+x_{2}$ steps).

The two parts are illustrated in Figure 3. The second part is composed of a sojourn of any length in states with zeros reward followed by sojourn of length 1 in a state with reward rate $f+e$ during which the process reaches level $k+e$ in a single step.

The theorem follows from (17) by applying matrix notation and the formula $\left(x_{1}+x_{2}\right)^{m}=\sum_{i=0}^{m}\left(\begin{array}{c}m \\ i\end{array}\right) x_{1}^{i} x_{2}^{m-i}$.

The following theorem is the "backward" counterpart of Theorem 5.

Theorem 6. The moments of the completion time with a given amount of excess, $e, 0 \leq e \leq c-1$, satisfies the backward recursion

$$
\mathbf{L}^{(m)}(k, e)=\sum_{i=0}^{m} \sum_{f=0}^{c-1}\left(\begin{array}{c}
m \\
i
\end{array}\right) \mathbf{L}^{(m-i)}(1, f) \mathbf{L}^{(i)}(k-f-1, e)
$$


Proof. The proof follows the line of the proof of Theorem 5.

In order to carry out the computations we need the matrices that provide the base cases of the recursion. Some of these matrices are trivial to compute and for what concerns these we have:

$$
\begin{aligned}
& \mathbf{L}^{(0)}(k, e)= \begin{cases}\mathbf{I} & \text { if } k \leq 0, k=-e \\
\mathbf{Z} & \text { if } k \leq 0, k \neq-e\end{cases} \\
& \mathbf{L}^{(i)}(k, e)=\mathbf{Z} \quad \text { if } i \geq 1, k \leq 0
\end{aligned}
$$

where negative values of $k$ are considered for computational convenience of the recursion.

Apart of the matrices listed above we need to compute $\mathbf{L}^{(i)}(1, e)$ with $i \geq 0$ and $0 \leq e \leq c-1$. To this end, it is useful to introduce the matrices $\mathbf{P}^{(0, i)}$ with $0 \leq i \leq c$ with entries according to

$$
\mathbf{P}_{k l}^{(0, i)}= \begin{cases}\mathbf{P}_{k l} & \text { if } k \in S_{0} \text { and } l \in S_{i} \\ 0 & \text { otherwise. }\end{cases}
$$

With these matrices, we have

$$
\mathbf{L}^{(i)}(1, e)=\sum_{k=1}^{\infty}(k+1)^{i}\left(\mathbf{P}^{(0,0)}\right)^{k-1} \mathbf{P}^{(0, e+1)} \mathbf{P}^{(e+1)}+\mathbf{P}^{(e+1)}
$$

where the first term of the right hand side considers the cases in which the process starts in a state with zero reward, it stays in the subset of states $S_{0}$ for $k-1$ steps, then it jumps to a state in $S_{e+1}$ and reaches level 1 with excess $e$ in the subsequent step. The second term considers instead the cases in which the process starts in a state belonging to $S_{e+1}$ and it reaches level 1 with excess $e$ in a single step.

In order to compute $\mathbf{L}^{(i)}(1, e)$ let us denote the summation in (18) by

$$
\mathbf{R}^{(i)}=\sum_{k=1}^{\infty}(k+1)^{i}\left(\mathbf{P}^{(0,0)}\right)^{k-1}
$$

By noting that

$$
\begin{aligned}
\mathbf{R}^{(i)} & =\sum_{l=0}^{i}\left(\begin{array}{l}
i \\
l
\end{array}\right) \sum_{k=1}^{\infty} k^{i-l}\left(\left(\mathbf{P}^{(0,0)}\right)^{k-1}\right) \\
& =\sum_{l=0}^{i}\left(\begin{array}{l}
i \\
l
\end{array}\right) \sum_{k=2}^{\infty} k^{i-l}\left(\left(\mathbf{P}^{(0,0)}\right)^{k-2} \mathbf{P}^{(0,0)}+\mathbf{I}\right) \\
& =\sum_{l=0}^{i}\left(\begin{array}{l}
i \\
l
\end{array}\right)\left(\mathbf{R}^{(i-l)} \mathbf{P}^{(0,0)}+\mathbf{I}\right)
\end{aligned}
$$

a recursive formula for $\mathbf{R}^{(i)}$ follows

$$
\mathbf{R}^{(i)}=\left[\sum_{l=1}^{i}\left(\begin{array}{l}
i \\
l
\end{array}\right)\left(\mathbf{R}^{(i-l)} \mathbf{P}^{(0,0)}+\mathbf{I}\right)+\mathbf{I}\right]\left(\mathbf{I}-\mathbf{P}^{(0,0)}\right)^{-1}
$$

The above expression points out that, as in case of continuous time MRMs, the presence of many states with zero reward increases the computational complexity. Indeed, in order to compute the $\mathbf{R}^{(i)}$ matrices, the inversion of a matrix with as many rows as many zero states there are is required. This matrix inversion leads to a full matrix and hence can increase drastically the space complexity as well. We briefly mention that, if a vector based implementation is applied then the matrix inversion can be avoided but the calculations would still require the solution of linear systems during every step of the computation.

The expressions given in Theorems 5 and 6 directly provide a procedure for the calculation of the moments of the completion time. If the number of zero states is low then analyzing the moments of the completion time is as complex as analyzing the moments of the aggregated reward.

The vector based application of Theorems 5 and 6 follows the same line as described for the accumulated reward at the end of Section 2.2.1. 


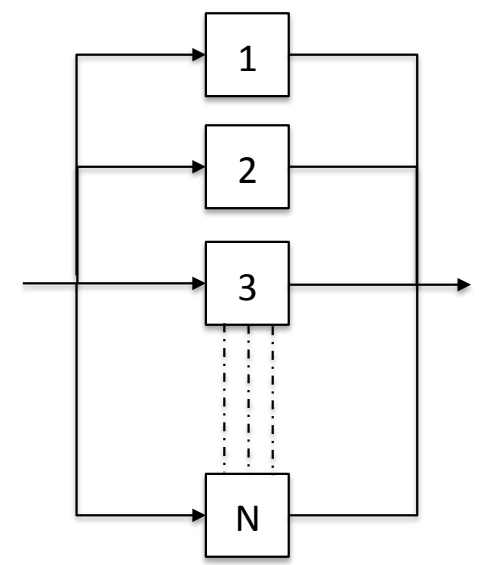

Figure 4: Layout of the system with $N$ identical parallel machines.

\section{Analysis of Systems by Markov Reward Models}

Due to the generality of the proposed approach, several system architectures and machine models can be analyzed within the framework described in the previous sections, both under the continuos and discrete assumptions $^{1}$. However, due to space limitations, in this paper we report the detailed analysis and results only for a few system architectures, including serial-parallel systems with limited repair capacity, buffered systems with degrading machines, and buffered systems producing in batches. The output moments of such systems have never been previously analyzed in the literature. Moreover, we focus on more traditional serial multi-stage manufacturing lines and we show the effect of down time distributions on the moment of the output performance. In addition, we derive some useful properties of this class of systems. For each example, the system configuration is represented by adopting the following formalism: machines are represented with squares, finite capacity buffers are represented with circles, and material flows are represented with arrows. All the numerical experiments reported in this section have been executed on a $2 \mathrm{GHz}$ Intel Centrino Dual Core, with $4 \mathrm{~Gb}$ of RAM.

\subsection{Parallel Machines with Limited Repair Capacity}

Consider a manufacturing system, or a portion of it, composed by $N$ identical parallel machines, as represented in Figure 4, each one characterized by failure rate $\lambda$, repair rate $\mu$ and attended by $K \leq N$ repairmen. If operational, each machine is always working and its processing rate is equal to 1 . If failed, a machine requires service by an operator to be restored to its operational condition. Within this experiment the impact of the number of repairmen on the moments of the system completion time and the cumulative production is investigated for the first time. The associated continuous MRM has the following infinitesimal generator (where we omit the diagonal entries)

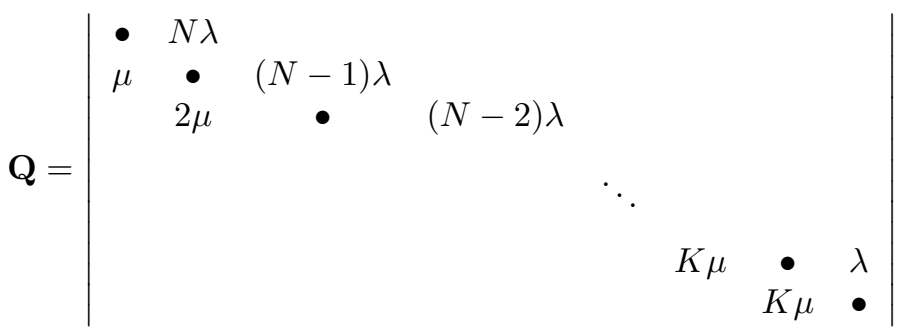

\footnotetext{
${ }^{1}$ In reference to the discrete time setting, a graphical JAVA tool for the computation of accumulated reward and completion time is available at http://www.di.unito.it/ $\sim$ angius .
} 

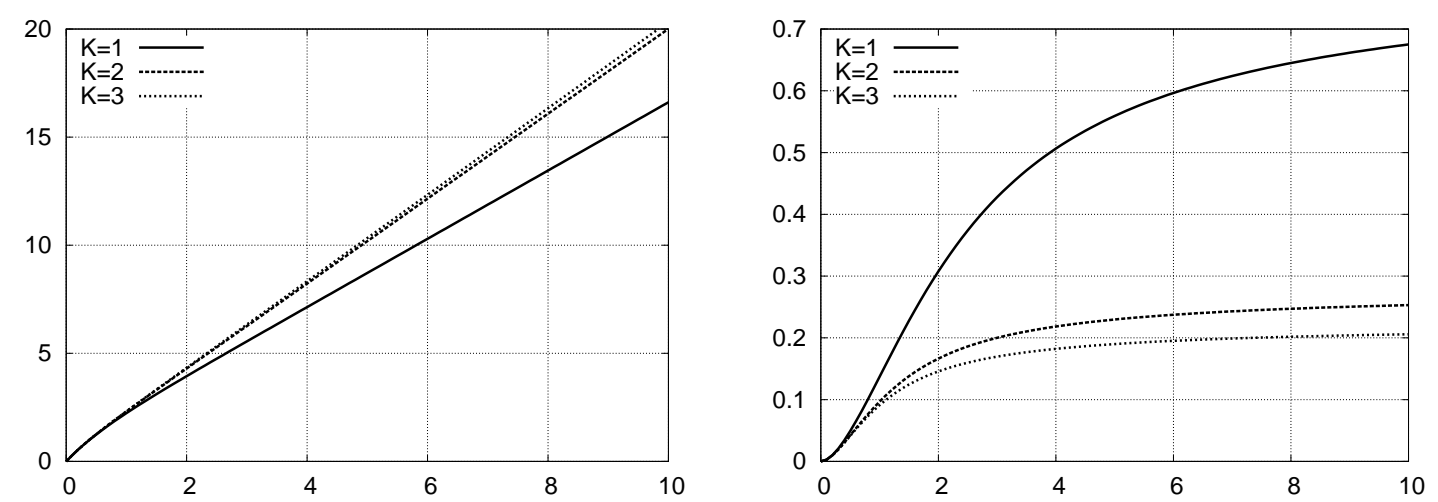

Figure 5: Accumulated reward as function of time for identical machines with limited repair capacity with $\lambda=1, \mu=2, N=3$ for different values of $K$ : mean (left) and index of dispersion (right)

and the matrix composed of the reward rates is

$$
\mathbf{R}=\left|\begin{array}{lllll}
N & & & & \\
& N-1 & & & \\
& & \ddots & & \\
& & & 1 & 0
\end{array}\right|
$$

The matrices $\mathbf{Q}$ and $\mathbf{R}$ of this simple model are such that the expressions given in (3) and (4) can be computed in a symbolic manner for any value of $N$ and $K$. This gives the possibility of analysing the production as function of $\lambda$ and $\mu$. Based on the properties of the Laplace transform, the Laplace transform of the moments of the accumulated reward (i.e., the Laplace transform of $\mathbf{K}^{(n)}(t)$ ) can be obtained from $\mathbf{F}^{* *}(s, v)$ as

$$
\mathbf{K}^{(n) *}(s)=\int_{t=0}^{\infty} e^{-s t} \mathbf{K}^{(n)}(t) d t=\lim _{v \rightarrow 0}(-1)^{n} \frac{d^{n} v \mathbf{F}^{* *}(s, v)}{d v^{n}}
$$

In general it is possible to obtain the inverse Laplace transform of the entries of $\mathbf{K}^{(n) *}(s)$ only if the numerical values of $\lambda, \mu, N$ and $K$ are known. For example, with $\lambda=1, \mu=2, N=3, K=2$ the mean accumulated reward at time $t$ given that the system is started with all machines up (obtained by summing up the entries of the first row of $\mathbf{K}^{(1) *}(s)$ and applying inverse Laplace transform) is

$$
0.3709+1.963 t+0.001644 e^{-8.372 t}-0.03 e^{-5 . t}-0.3425 e^{-2.627 t}
$$

while the second moment (obtained the same way from $\mathbf{K}^{(2) *}(s)$ ) is

$$
\begin{gathered}
-0.2429+2.0009 t+3.8558 t^{2}+0.004623 e^{-8.372 t} t-0.084 e^{-5 . t} t \\
-0.8434 e^{-2.627 t} t+0.003189 e^{-8.372 t}+0.1776 e^{-5 . t}+0.06217 e^{-2.627 t} .
\end{gathered}
$$

In Figure 5 the mean and the index of dispersion of the cumulated production as function of time are depicted. While the cases $K=2$ and $K=3$ are almost identical for what concerns the mean, they differ for the variance of the cumulated production. Therefore, by only looking at the first moment of the cumulated production, one would probably take the decision of dedicating 2 repairmen to this system, since the additional personnel cost is not compensated by a significant throughput gain. However, by also considering the second moment, the best decision could be allocating one additional repairmen to reduce the variance of the output of a $20 \%$, for example at time 10 , thus generating a more stable output, ultimately compensating the additional personnel cost by a reduction of backlog costs. 
In general, it is not possible to obtain expressions for the moments of the production as function of time and the parameters of the model $(\lambda, \mu, N, K)$ but a symbolic expression for the asymptotic behaviour of these functions can be obtained. This can be done as follows. The non-vanishing terms of the mean are of the form $a_{10}+a_{11} t$ while for the second moment it is $a_{20}+a_{21} t+a_{22} t^{2}$. Denoting by $m_{1}(t)$ and $m_{2}(t)$ the mean and the second moment of the accumulated reward at time $t$ and by $m_{1}^{*}(s)$ and $m_{2}^{*}(s)$ their Laplace transforms (which can be easily obtained by appropriate summations of the entries of $\mathbf{K}^{(1) *}(s)$ and $\mathbf{K}^{(2) *}(s)$ ), the coefficients describing the non-vanishing terms can be obtained as

$$
\begin{aligned}
& a_{11}=\lim _{s \rightarrow 0} s^{2} m_{1}^{*}(s) \\
& a_{10}=\lim _{s \rightarrow 0} s\left(m_{1}^{*}(s)-\frac{a_{11}}{s^{2}}\right) \\
& a_{22}=\frac{1}{2} \lim _{s \rightarrow 0} s^{3} m_{2}^{*}(s) \\
& a_{21}=\lim _{s \rightarrow 0} s^{2}\left(m_{2}^{*}(s)-\frac{2 a_{22}}{s^{3}}\right) \\
& a_{20}=\lim _{s \rightarrow 0} s\left(m_{2}^{*}(s)-\frac{2 a_{22}}{s^{3}}-\frac{a_{21}}{s^{2}}\right) .
\end{aligned}
$$

Having obtained the above coefficients the limiting value of the index of dispersion can be obtained as

$$
I=\frac{a_{21}-2 a_{11} a_{10}}{a_{11}} .
$$

The above procedure provides symbolic expressions for any value of $N$ and $K$. For example, with $N=3$ we have that the limiting values of the index of dispersion is

$$
\begin{aligned}
& \frac{2 \lambda\left(72 \lambda^{6}+216 \mu \lambda^{5}+324 \mu^{2} \lambda^{4}+240 \mu^{3} \lambda^{3}+94 \mu^{4} \lambda^{2}+16 \mu^{5} \lambda+\mu^{6}\right)}{\left(2 \lambda^{2}+2 \mu \lambda+\mu^{2}\right)\left(6 \lambda^{3}+6 \mu \lambda^{2}+3 \mu^{2} \lambda+\mu^{3}\right)^{2}} \\
& \frac{\lambda\left(18 \lambda^{6}+108 \mu \lambda^{5}+234 \mu^{2} \lambda^{4}+249 \mu^{3} \lambda^{3}+146 \mu^{4} \lambda^{2}+48 \mu^{5} \lambda+8 \mu^{6}\right)}{(\lambda+\mu)^{2}\left(3 \lambda^{3}+6 \mu \lambda^{2}+6 \mu^{2} \lambda+2 \mu^{3}\right)^{2}} \\
& \frac{2 \lambda}{(\lambda+\mu)^{2}}
\end{aligned}
$$

for $K=1,2$ and 3, respectively, where the simplicity of the last expression is due to the fact that with $K=3$ the model simplifies to the sum of three independent machines.

Now we turn our attention to the analysis of the completion time. The same steps we have applied to the double transform of the accumulated reward, $\mathbf{F}^{* *}(s, v)$, can be applied to the double transform of the completion time, $\mathbf{G}^{* *}(s, v)$. We report briefly the results that can be obtained. With $\lambda=1, \mu=2, N=$ $3, K=2$, the mean of the completion time for $x$ units of product is

$$
-0.1183+0.5092 x-0.006899 e^{-5.302 x}+0.1252 e^{-1.697 x}
$$

while its second moment is

$$
\begin{aligned}
& -0.05676-0.04862 x+0.2593 x^{2}-0.01401 e^{-5.302 x} x+ \\
& 0.1398 e^{-1.697 x} x-0.005304 e^{-5.302 x}+0.06207 e^{-1.697 x} .
\end{aligned}
$$

Figure 6 provides the mean and the index of dispersion of the completion time as function of the required quantity of the product. The same phenomenon can be observed as for the accumulated reward: the mean completion time is almost identical for the cases $K=2$ and $K=3$ but some difference occurs between these two cases for what concerns the variability of the completion time. 

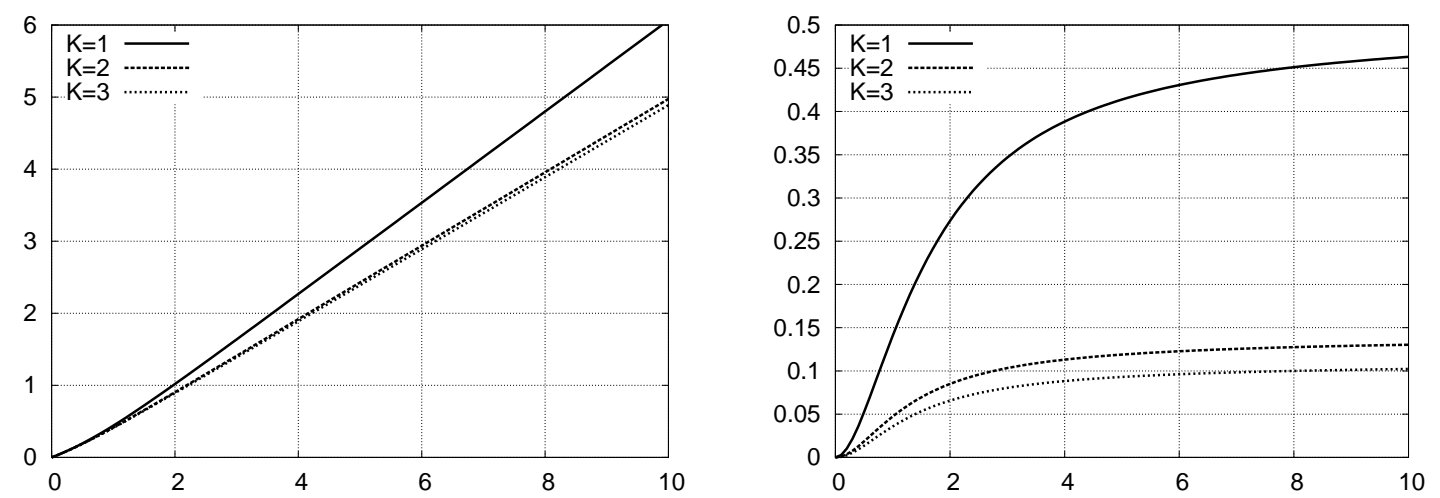

Figure 6: Completion time as function of the required quantity of the product for identical machines with limited repair capacity with $\lambda=1, \mu=2, N=3$ for different values of $K$ : mean (left) and index of dispersion (right)

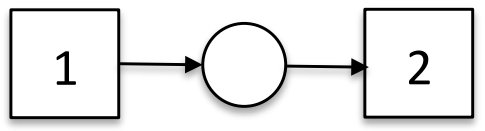

Figure 7: Layout of the buffered two-machine line.

The limiting index of dispersion of the completion time can be obtained without assigning numerical values to $\lambda$ and $\mu$, and with $N=3$ we have

$$
\begin{aligned}
& \frac{2 \lambda\left(72 \lambda^{6}+216 \mu \lambda^{5}+324 \mu^{2} \lambda^{4}+240 \mu^{3} \lambda^{3}+94 \mu^{4} \lambda^{2}+16 \mu^{5} \lambda+\mu^{6}\right)}{3 \mu\left(2 \lambda^{2}+2 \mu \lambda+\mu^{2}\right)^{2}\left(6 \lambda^{3}+6 \mu \lambda^{2}+3 \mu^{2} \lambda+\mu^{3}\right)} \\
& \frac{\lambda\left(18 \lambda^{6}+108 \mu \lambda^{5}+234 \mu^{2} \lambda^{4}+249 \mu^{3} \lambda^{3}+146 \mu^{4} \lambda^{2}+48 \mu^{5} \lambda+8 \mu^{6}\right)}{6 \mu(\lambda+\mu)^{4}\left(3 \lambda^{3}+6 \mu \lambda^{2}+6 \mu^{2} \lambda+2 \mu^{3}\right)} \\
& \frac{2 \lambda}{3 \mu(\lambda+\mu)}
\end{aligned}
$$

for $K=1,2$ and 3 , respectively,

The procedure we have illustrated here for parallel machines with limited repair capacity with continuous time and continuous product can be performed with discrete time and discrete product as well by applying basic properties of z-transform.

\subsection{Discrete Buffered Two-machine Line with Degrading Machines}

As a second example, we analyse the output of the second machine of a buffered two-machine line characterized by machines that undergo a deterioration process. The main goal of this experiment is to investigate the impact of the buffer size on the moments of the performance measures, for these previously uninvestigated system features. The system is represented in Figure 7. We assume discrete time and production of discrete quantities. Moreover, we assume that the two machines are identical and operate according to a degradation/reparation scheme. The underlying DTMC modeling the dynamics of each 
machine has the following transition probability matrix:

$$
\mathbf{P}=\left|\begin{array}{cccccccc}
1-p & p & & & & & & \\
& 1-p & p & & & & & \\
& & \ddots & & & & & \\
& & & 1-p & p & & & \\
& & & & 1-q & q & & \\
& & & & & 1-q & q & \\
& & & & & & \ddots & \\
q & & & & & & & 1-q
\end{array}\right|
$$

with $N_{u}$ up-states and $N_{d}$ down-states. We assume that the quantity produced in the up-states is $N_{u}, N_{u}-$ $1, N_{u}-2, \ldots, 2,1$, i.e., the productivity of the machines degrades as it crosses the up-states. There is no production in the down-states. Therefore, at the end of each time slot, the first machine delivers $r_{i}$ parts in the buffer (if it is in state $i$ ) and then the second machine extracts $r_{j}$ parts from the buffer (if it is in state $j$ ). When the buffer is totally full the first machine is blocked and it is not allowed to change state; the same happens to the second machine when the buffer is totally empty (Operation Dependent Transitions). When the buffer is close to being full (empty) then the first (second) machine produces as much as allowed by the current buffer content (by the current available parts). This assumption is also sometimes referred as "partial batching". Under these assumptions, it is straightforward to build a DTMC describing the dynamics of the whole system, which accounts for both the states of the two machines and the number of parts stored in the buffer. In detail, the state of the system is described by the triple $\left(\alpha_{1}, \alpha_{2}, b\right)$, where $\alpha_{1}$ refers to the state of the first machine, $\alpha_{2}$ refers to the state of the second machine and the buffer level $b$ assumes values $0,1,2, \ldots, B$. The machines act as independent in internal states $(0<b<B)$ and the transitions between the system states are obtained by multiplying the corresponding transitions of the DTMC describing the behavior of each machine in isolation. At the boundary $(b=0, b=B)$, due to the Operation Dependent Transitions assumption, the transitions are only determined by the failed machine that generates the starvation or blocking event. The output reward is obtained by considering the states where the second machine is operational and not starved.

For this system, considering empty buffer and fully operational machines as initial state, we compute the mean and the index of dispersion of the number of parts produced by the second machine by applying the recursion given in (13) for $N_{u}=10, N_{d}=5, p=0.1, q=0.5$, under variation of the buffer size $B$. The results are depicted in Figure 8 and Figure 9. As expected, the mean cumulative production is positively affected by the buffer. Indeed, higher buffer results in higher mean cumulated production. For example, if 2800 parts have to be delivered before time 800 a buffer size of 10 shall be selected, if the first moment of the production output is observed. However, if the index of dispersion of the cumulated production is observed, a more complex behavior is captured. Indeed, the index of dispersion is low for low buffer size $(B=5)$, then drastically increases with medium buffer size $(B=10)$ and finally decreases for increasing buffer size $(B=50, B=100)$. Therefore, the configuration with $B=10$ provides an index of dispersion of the cumulated production that is about three times larger than the index of dispersion with $B=5$. Therefore, by moving from a configuration with $B=10$ to a configuration with $B=5$ the output stability can be consistently increased at the cost of reducing the mean cumulated production. The oscillatory behaviour of the variability as a function of time is due to the fact that both the time to complete failure and the time to repair of the machines are of low variance. The size of the state space is given by $\left(N_{u}+N_{d}\right)^{2}(B+1)$ which in our case is 22725 for $B=100$. Note that the method is applicable for much larger state spaces as well. 

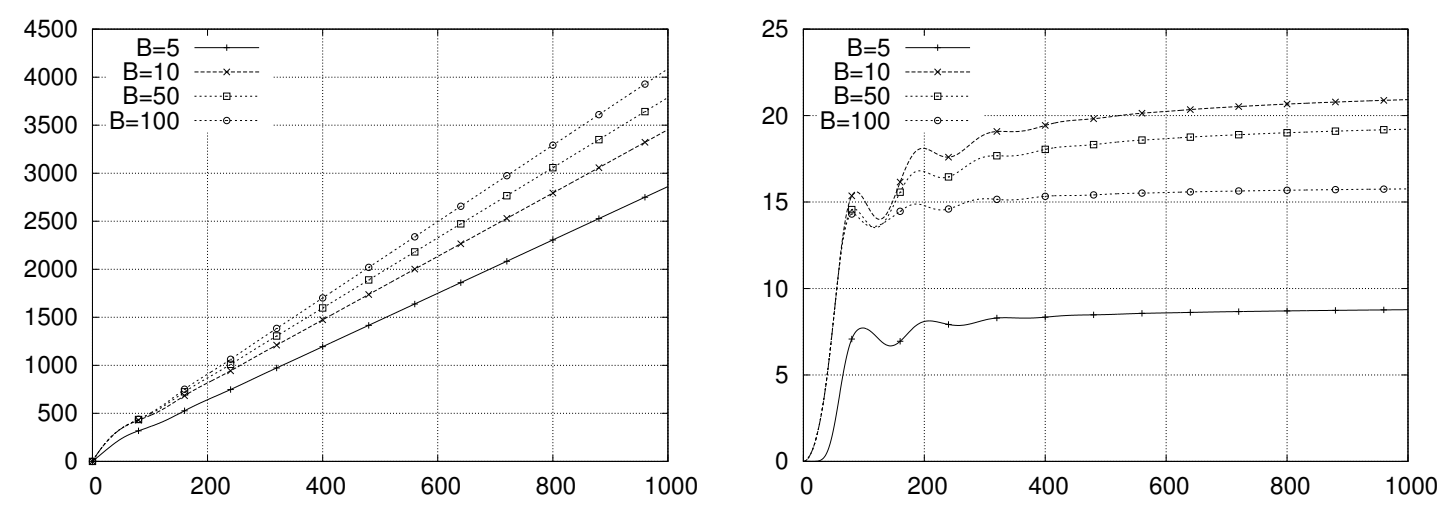

Figure 8: Number of produced parts as function of time for the machine-buffer-machine block with different buffer sizes: mean (left) and index of Dispersion (right)
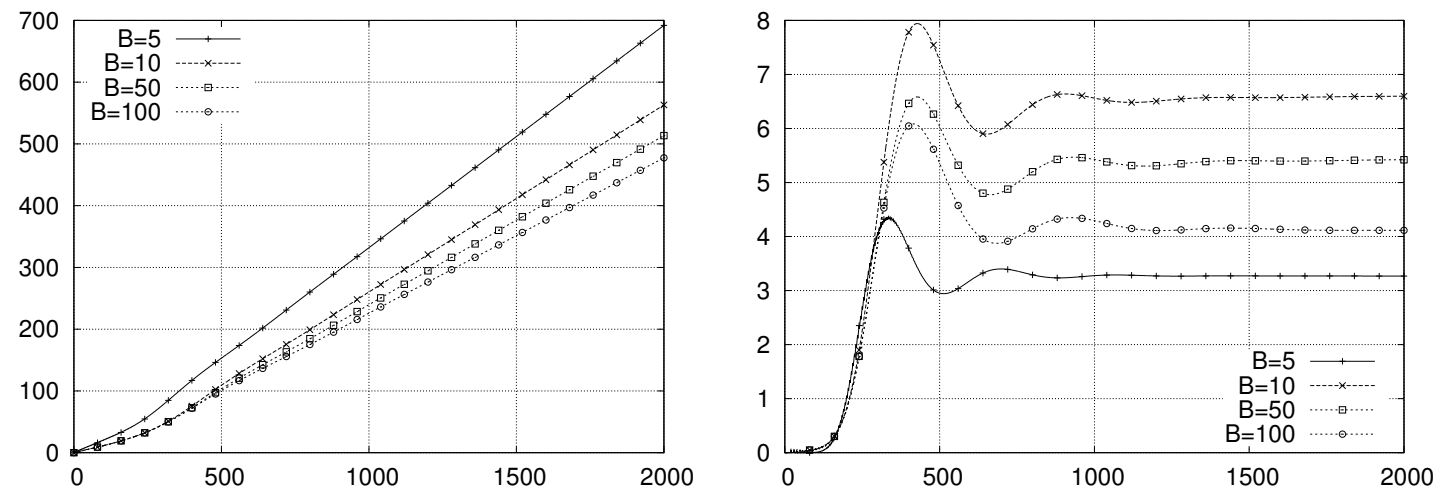

Figure 9: Time required to complete a task as function of the number of items for the machine-buffer-machine block with different buffer sizes : mean (left) and variance (right)

\subsubsection{Skewness and higher order moments}

As third test, we illustrate the use of higher order moments in the analysis of a machine-buffer-machine block. We assume that both machines have geometric failure times with mean time to failure equal to 100. Also for what concerns times to repair we assume that the two machines are identical but we consider three different distributions with mean equal to 10: the geometric distribution, the order two hyper-geometric distribution with coefficient of variation (CV) equal to 2, and the order two hypo-geometric distribution with $\mathrm{CV}$ equal to 0.5 . Both machines produce one part per time slot in the up state. The capacity of the buffer is 5 .

The idea behind these tests is to refute the hypothesis that the normal distribution is a good estimator of the distribution of the number of items produced at a given time unit. This has been done by estimating the symmetry of the distribution through the computation of the skewness ${ }^{2}$. If the service level of the lines was following a normal distribution then the skewness of both the accumulated reward and the completion time would be constant to zero. Figure 10 confirms our belief, i.e., in a short time horizon, the hypothesis above is a biased estimator. This is showed on the r.h.s. where all the three curves of the skewness start quite far from zero and get reasonably close to zero after time 2000 only.

\footnotetext{
${ }^{2}$ The skewness corresponds to the standardized third moment and is equal to $\frac{E\left[(X-\mu)^{3}\right]}{E\left[(X-\mu)^{2}\right]^{3 / 2}}$
} 

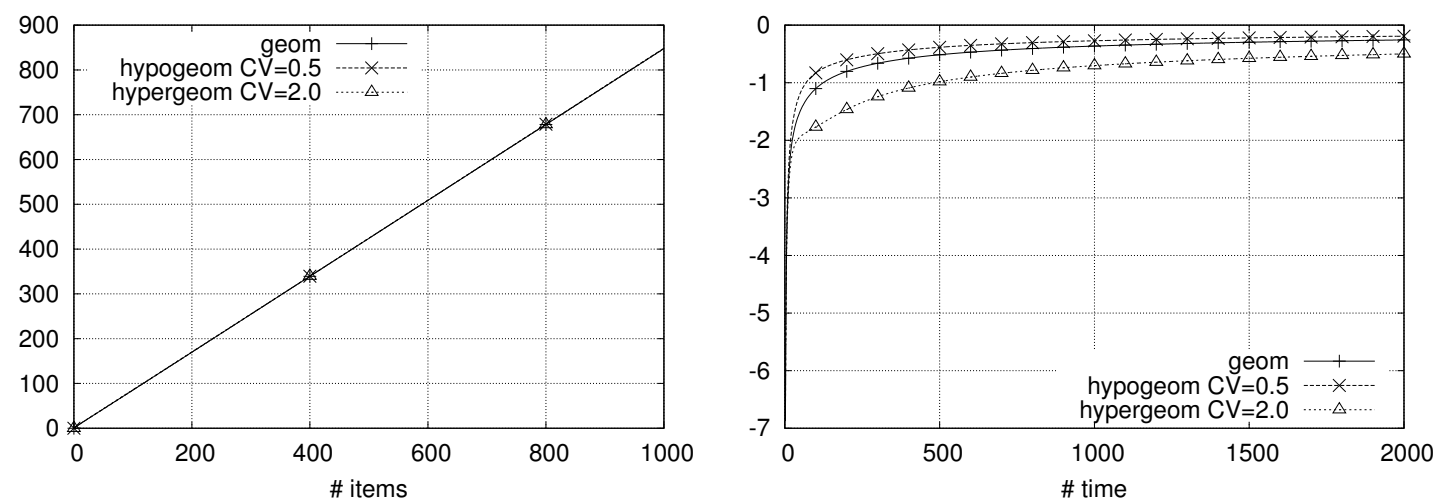

Figure 10: Number of produced parts as function of time for the machine-buffer-machine blocks with different coefficients of variation: mean (left) and skewness (right)

Figure 11 gives a quantification of the error in regards to the size of the lot. In particular, we can observe that the error can be consistent for small and medium lot sizes. Thus, the normal distribution assumption overestimates the service level leading to the risk of generating optimistic system configurations.
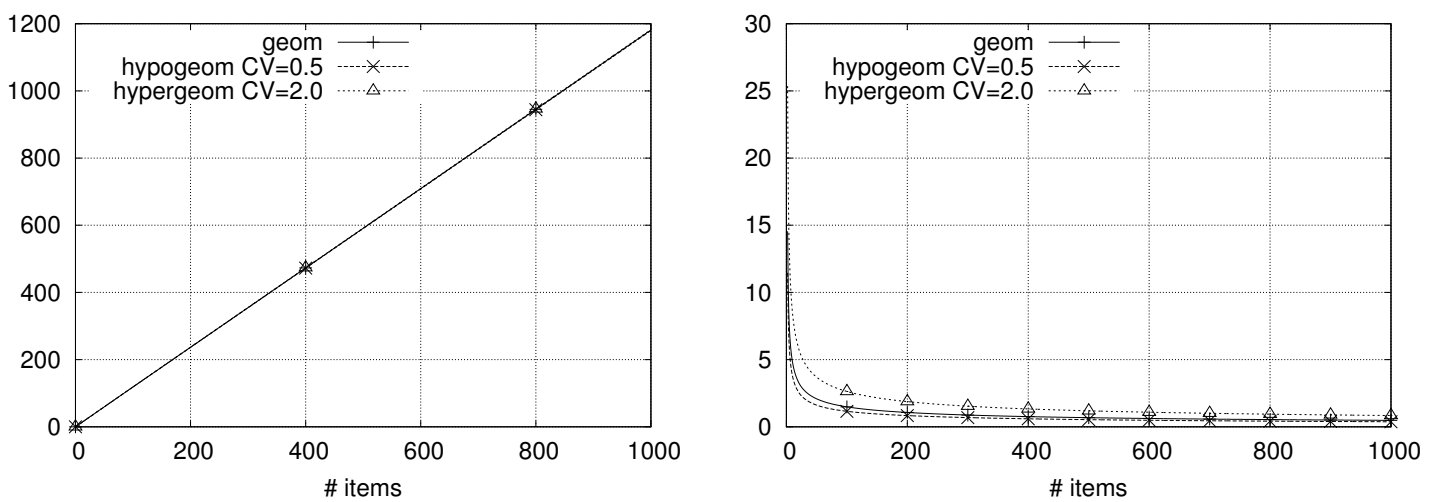

Figure 11: Time required to complete a task as function of the number of items for the machine-buffer-machine block with different coefficients of variation : mean (left) and skewness (right)

On the left side of the figures it is possible to observe that as far as the mean sojourn times are the same, the expected service level is independent from the CVs.

\subsection{Systems with Machines Producing in Batches}

The fourth set of tests deals with the impact of batch productions in case of full batching policy ([3]). The main goal of this experiment is to investigate the impact of the batch size on the moments of the performance measures.

We consider a two machine system, with machines having failure and repair times distributed according a geometric distribution, with mean as in the previous test case. The size of the buffer is set to 20. Full batching means that the upstream machine can only produce a batch if there is enough space in the downstream buffer. Similarly, the downstream machine can only produce a batch if there are enough semi-finished parts in the upstream buffer.

We tested different batch sizes and assumed two scenarios: in the first, the upstream machine produces only in batch, whereas the downstream machine is able to produce only one item per unit time, if operational; 
in the second scenario, the situation is reversed. Figures 12 and 13 depict both the mean and the index of dispersion of the cumulated production for the first scenario. It is possible to observe that the variability of the cumulated production decreases by increasing the batch size of the first machine although the expected production remains almost unchanged.
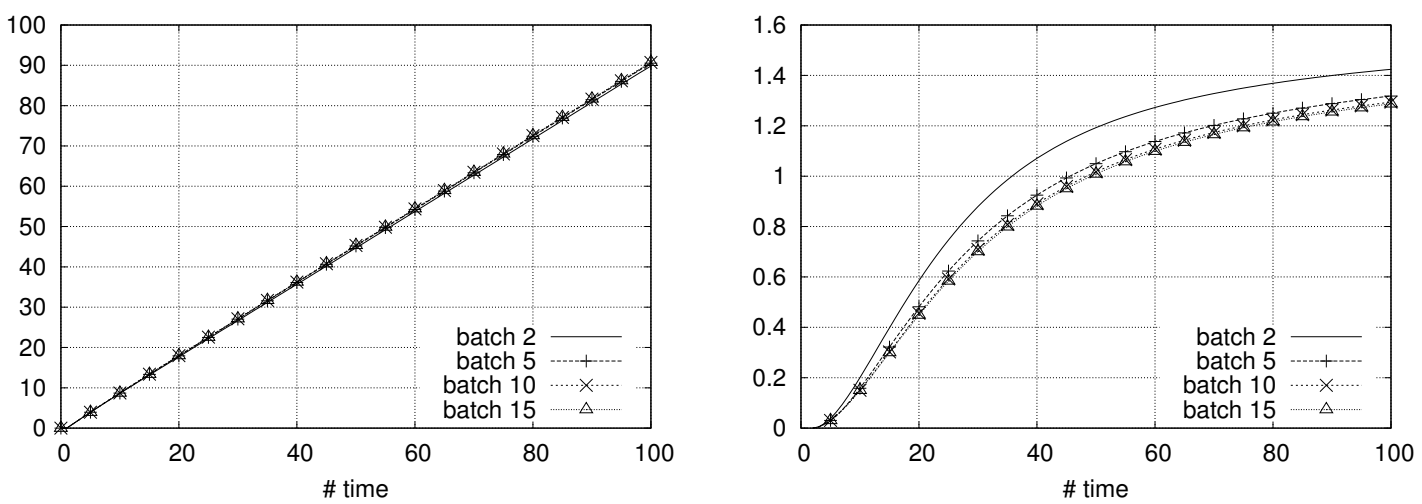

Figure 12: Number of produced parts as function of time for the machine-buffer-machine block with different batch sizes on the first machine : mean (left) and index of dispersion (right)
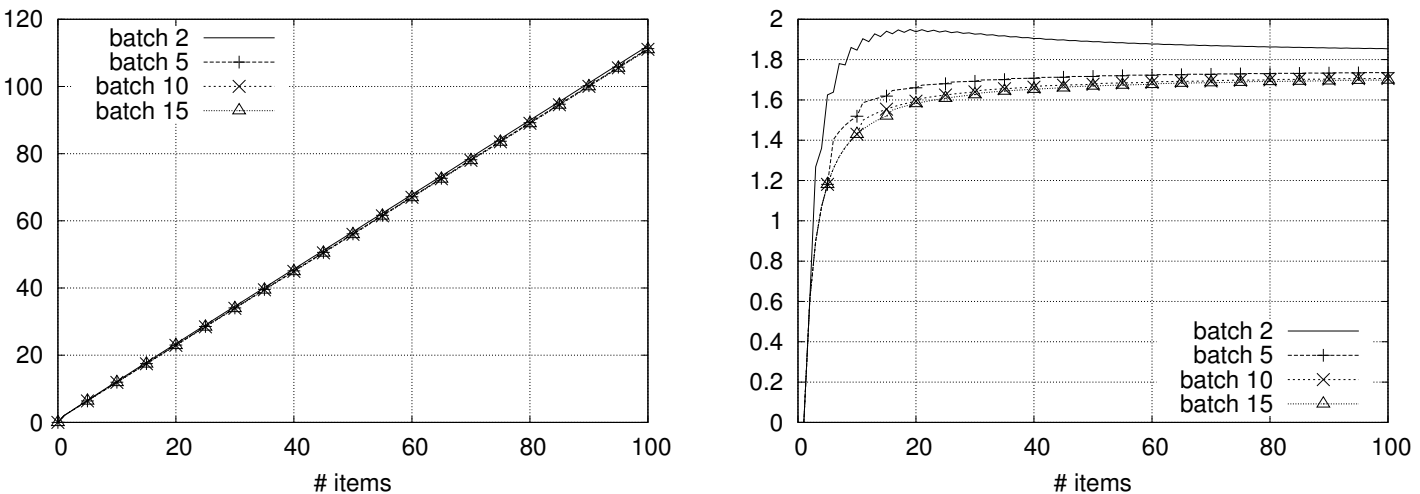

Figure 13: Time required to complete a task as function of the number of items for the machine-buffer-machine block with different batch sizes on the first machine : mean (left) and index of dispersion (right)

The results for the second scenario are depicted in Figures 14 and 15. The figures show that, the expected cumulated production quantity is characterized by step curves. In particular, the curves show the presence of cold points (minimal result with maximum effort) and hot points (maximum result with the minimal effort) both for short time intervals and for small lot sizes. For example, assuming a batch size equal to 15, the production of a lot of 16 items requires the same time as the production of a lot composed of 30 parts. This analysis may help the decision about lot sizing, taking into account both the mean and the variance of the cumulated production.

The right sides of the figures show the variability of the cumulated production. It is possible to observe that the larger the batch and the more variability is observed in the cumulated production. In particular, much larger is the dimension of the batch and much larger are the spikes that characterize the accumulate reward index of dispersion. 


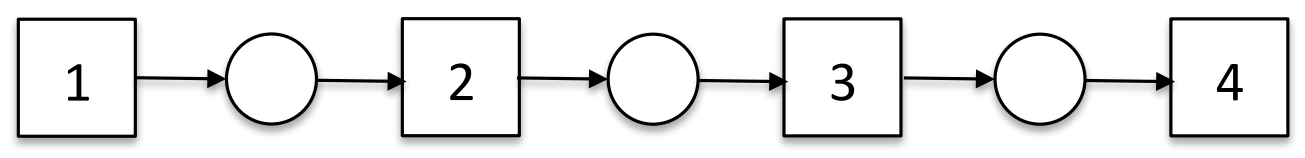

Figure 16: Layout of the considered four-machine line.
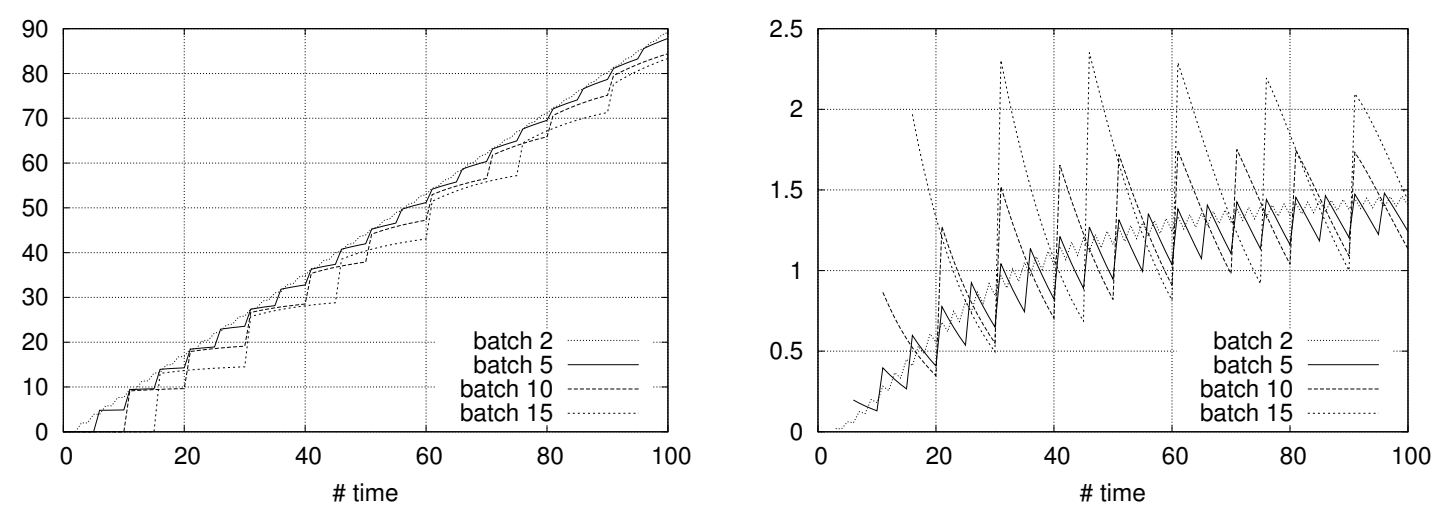

Figure 14: Number of produced parts as function of time for the machine-buffer-machine block with different batch sizes on the second machine : mean (left) and index of dispersion (right)
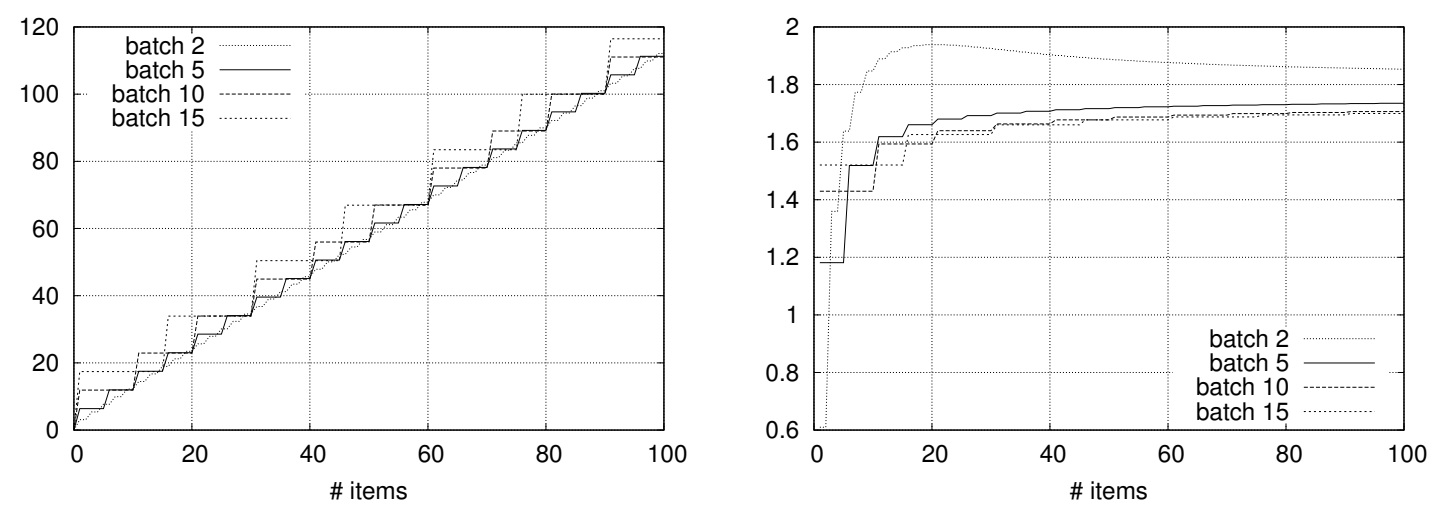

Figure 15: Time required to complete a task as function of the number of items for the machine-buffer-machine block with different batch sizes on the second machine : mean (left) and index of dispersion (right)

From this simple example it can be concluded that the reversibility property does not hold for batch production, since the system and its reversed system show different behavior, both in terms of mean and variance of the cumulated production.

\subsection{Multi-stage Buffered Systems with Single Up-down Machines}

\subsubsection{Reversibility Property for the Asymptotic Second Moments}

The following tests deal with production lines formed by four machines, Figure 16, each one having geometric failure and repair times with mean equal to 100 and 10, respectively. We test the effect of different buffer sizes on the output variability. 
The idea behind the test is to show that the reversibility property holds also for higher order performance measures. Indeed, It was proved in [24] that, in buffered production lines, the average throughput remains invariant under reversal of the line. Line reversal means that every item passes through the stations in the reverse order, that is, beginning with the last station and ending with the first station. However, this property has never been discussed for higher order performance measures. In our example, since all machines are identical, this means that a production line having three buffers with capacity 5, 5, 2 provides the same cumulated output variance of a line whose buffers have been inverted, i.e. 2, 5, 5. In this section, we experimentally show that this property is always true when the time approaches infinity whereas in a finite time horizon it is true only if the machines start from the same state. Figures 17 and 18 illustrate these results. A formal proof of this statement will be subject of future works.
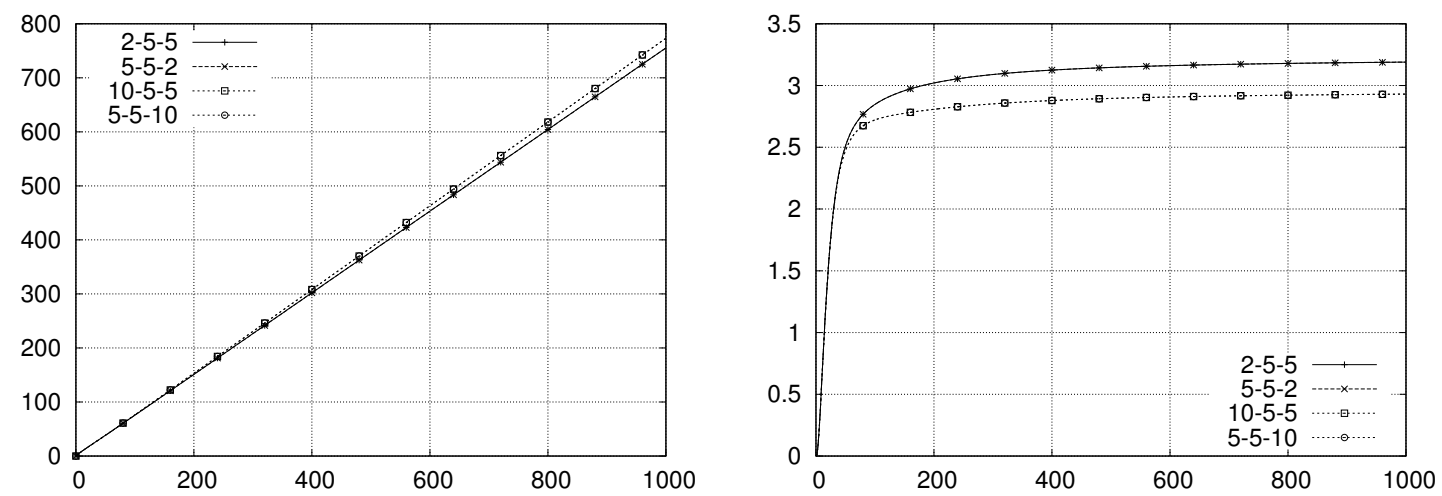

Figure 17: Number of produced parts as function of time for a production line composed of four machines and three buffers having sizes $B_{1}-B_{2}-B_{3}$ : mean (left) and index of dispersion (right)
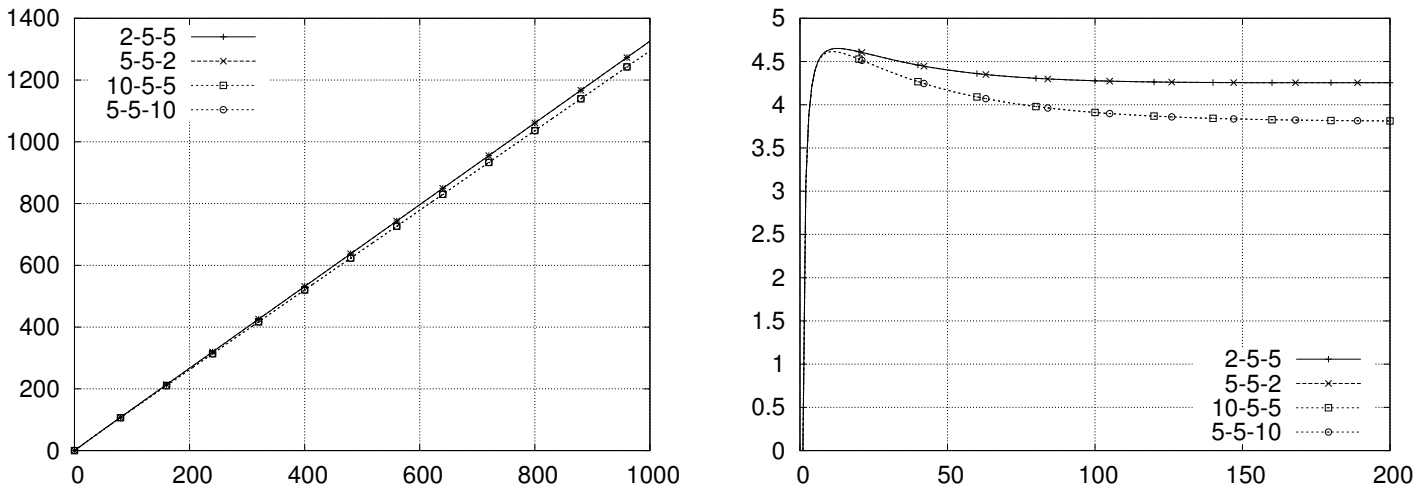

Figure 18: Time required to complete a task as function of the number of items for a production line composed of four machines and three buffers having sizes $B_{1}-B_{2}-B_{3}$ : mean (left) and index of dispersion (right)

\subsubsection{Six machines - Estimation completion time}

In this last experiment, we consider a Markovian model of the production line in Figure 19, composed of six machines and five buffers, each one with a capacity of 5 ; hence the whole state space is composed of $2^{6} \times 6^{5}=497664$ states. This model dimension does not allow the direct computation of the completion time distribution. The reason is that the number of states with zero production is high and the inversion of the matrix $\mathbf{P}^{(0,0)}$ generates a dense matrix whose handling is not possible. Therefore, we compute the completion time distribution using the duality with the accumulated reward distribution. Specifically, we 


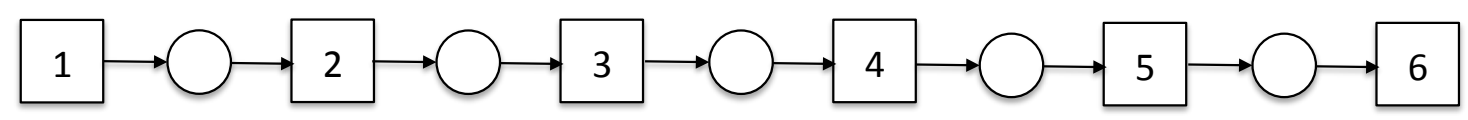

Figure 19: Layout of the considered six-machine line.

apply the proposed approach to compute the first 20 moments of the accumulated reward of the system. Then, we approximated the distribution of this measure by using the method described by Telek and Tari in $[15,33]$. Given a r.v. $X$, this method takes as input a finite number of moments of $X$ and returns an upper and lower bound for the probability $\operatorname{Pr}\{X=x\}$. The method works in such a way that the more $x$ is far from the expectation, the more accurate the bounds are. In general, the accuracy of the method (tighter intervals) improves if the number of moments increases. The computation of the 20 considered moments required half an hour. Note that the computation of the moments of the accumulated reward is feasible for bigger state spaces. The result are shown in Figures 20 and in Figure 21. In particular, Figures 20 shows the expected value and the index of dispersion of the cumulated reward since time 2000. Figure 21 shows the approximated completion time c.d.f. for a lot of 2000, in comparison with its normal approximation, obtained by considering only the first two moments. As it can be noticed, in this case the completion time c.d.f. calculated with the normal distribution approximation is within the bounds calculated with the method proposed in $[15,33]$. This is in line with the results reported in section 3.2 .1 , since the lot size in the considered case is large enough for accepting the normal distribution. Therefore, the method proposed in this paper can be used to approximate the entire distribution of the considered performance measures, enabling to accept or refuse the normal distribution approximation, depending on the considered system parameters and time or lot size.
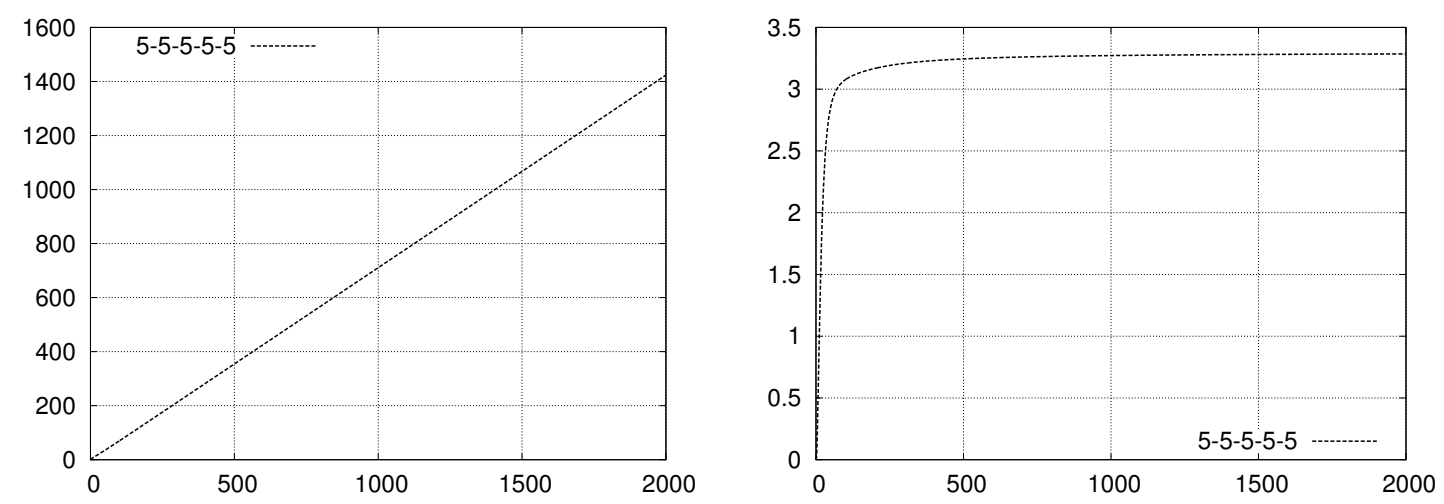

Figure 20: Number of produced parts as function of time for a six machine production line: mean (left) and index of dispersion (right) 


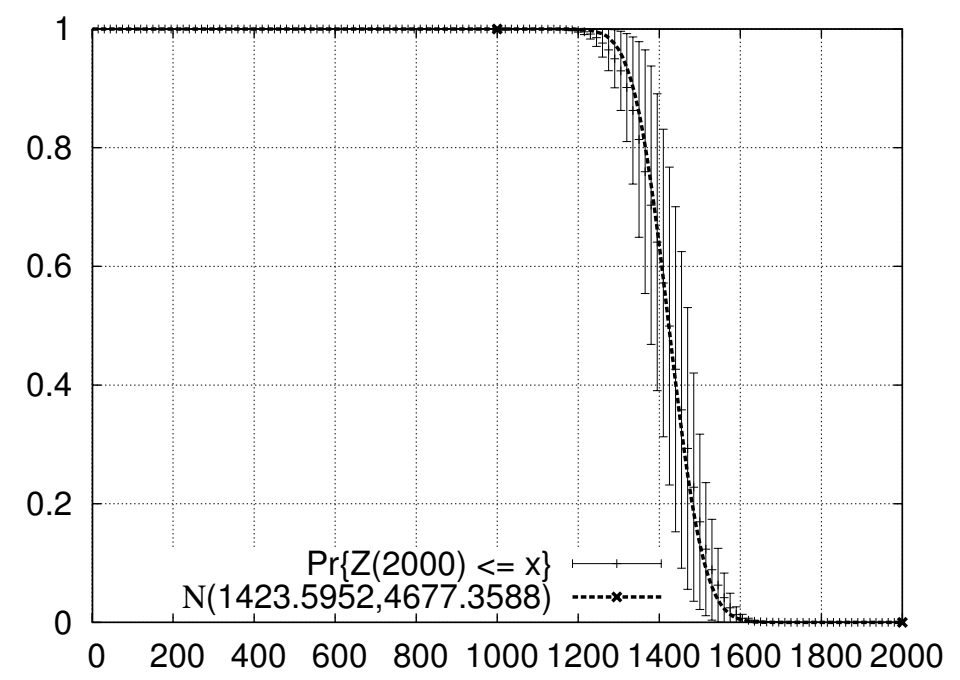

Figure 21: C.d.f. estimation of the completion time for a six machine production line

\section{Conclusions}

In this paper, a methodology for the analysis of the moments of the cumulated output and the completion time of unreliable Markovian systems has been presented. The framework is based on both continuous and discrete Markov reward models of general complexity, which allows for modeling several system architectures and failure/repair mechanisms. The approach allows the derivation of closed form formulas for the second moment of the production output for specific system settings. The main results reported in this paper are as follows:

- The stability of the output can be increased by changing the buffer size. In particular, there are cases where it is possible to reduce the variance of the cumulated output at the cost of reducing the mean of the cumulated output.

- The service level calculated by approximating the cumulated production by a normal distribution is a biased estimator. The error can be consistent, especially for small and medium lot sizes. In particular, this approximate service level estimator overestimates the service level, thus leading to the risk of generating optimistic system configurations.

- The system "reversibility" property holds for the asymptotic variance rate and the asymptotic completion time. It does not hold, in general, for the second moment of the cumulated production, at a given time, and the second moment of the completion time, at a given lot size.

- The number of operators in parallel machining stages consistently affects both the mean and the variance of the cumulated production. In particular, increasing the number of repairmen is beneficial since it increases the first moment and decreases the second moment of the system output.

Future research activities will be dedicated to the extension of the approach to deal with systems with multiple random rewards. This extension would allow including in the model the case of impulse type rewards. Moreover, particular attention will be given to the analysis of the transient period of production systems depending on the initial system conditions, since the presented analysis can be in principle applied to study this problem. Furthermore, more complex systems design problems will be tackled including the computation of higher moments of the performance measures directly in the problem formulation. 


\section{Acknowledgments}

This work has been supported in part by project "AMALFI - Advanced Methodologies for the Analysis and management of Future Internet" sponsored by Universitå di Torino and Compagnia di San Paolo.

\section{References}

[1] A. Bobbio, A. Horváth, M. Telek, Matching three moments with minimal acyclic phase type distributions, Stochastic models 21 (2-3) (2005) 303-326.

[2] M. Carrascosa, Variance of the output in a deterministic two-machine line, Master's thesis, Massachusetts Institute of Technology, Cambridge MA 02139 (1995).

[3] S. Chang, S. Gershwin, Modeling and analysis of two unreliable batch machines with a finite buffer in between, IIE Transactions 42 (6) (2010) 405-421.

[4] C.-T. Chen, J. Yuan, Transient throughput analysis for a series type system of machines in terms of alternating renewal processes, European Journal of Operational Research 155 (1) (2004) 178-197.

[5] P. Ciprut, M.-O. Hongler, Y. Salama, Fluctuations of the production output of transfer lines, Journal of Intelligent Manufacturing 11 (2000) 183-189, 10.1023/A:1008942917166.

[6] M. Colledani, M. Ekvall, T. Lundholm, P. Moriggi, A. Polato, T. Tolio, Analytical methods to support continuous improvements at Scania, International Journal of Production Research 48 (2010) 1913-1945.

[7] M. Colledani, A. Matta, T. Tolio, Analysis of the Production Variability in Manufacturing Lines, ASME Conference Proceedings 2008 (48357) (2008) 381-390.

[8] M. Colledani, A. Matta, T. Tolio, Analysis of the production variability in multi-stage manufacturing systems, CIRP Annals - Manufacturing Technology 59 (1) (2010) 449-452.

[9] C. Dincer, B. Deler, On the Distribution of Throughput of Transfer Lines, The Journal of the Operational Research Society 51 (10) (2000) 1170-1178.

[10] S. B. Gershwin, Variance of the Output of a Tandem Production System, in: R. D. Onvural, I. F. Akyildiz (eds.), Proceedings of the Second International Conference on Queuing Networks with Finite Capacity, 1993.

[11] S. B. Gershwin, Manufacturing Systems Engineering, PTR Prentice Hall, 1994.

[12] W. K. Grassman, Means and variances in Markov reward systems, in: C. D. Meyer, R. J. Plemmons (eds.), Linear Algebra, Markov Chains and Queueing Models, vol. 48 of The IMA Volumes in Mathematics and Its Applications, Springer-Verlag, New York, 1993, pp. 193-204.

[13] K. B. Hendricks, The Output Processes of Serial Production Lines of Exponential Machines with Finite Buffers, Operations Research 40 (6) (1992) 1139-1147.

[14] K. B. Hendricks, J. O. McClain, The Output Processes of Serial Production Lines of General Machines with Finite Buffers, Management Science 39 (10) (1993) 1194-1201.

[15] G. Horváth, S. Rácz, Á. Tari, M. Telek, Evaluation of Reward Analysis Methods with MRMSolve 2.0, in: QEST, 2004, pp. $165-174$.

[16] R. Inman, Empirical Evaluation of Exponential and Independence Assumptions in Queueing Models of Manufacturing Systems, Production Operations Management 8 (1999) 409-432.

[17] V. Kulkarni, V. Nicola, K. Trivedi, On Modeling the Performance and Reliability of Multi-Mode Computer Systems, The Journal of Systems and Software 6 (1986) 175-183.

[18] J. Li, S. Meerkov, Production variability in manufacturing systems: Bernoulli reliability case, Annals of Operations Research 93 (1) (2000) 299-324.

[19] J. Li, S. Meerkov, Production Systems Engineering, Springer, 2009.

[20] M. Manitz, H. Tempelmeier, The variance of inter-departure times of the output of an assembly line with finite buffers, converging flow of material, and general service times, OR Spectrum (2010) 1-19.

[21] S. Meerkov, N. Shimkin, L. Zhang, Transient Behavior of Two-Machine Geometric Production Lines, Automatic Control, IEEE Transactions on 55 (2) (2010) 453-458.

[22] G. J. Miltenburg, Variance of the number of units produced on a transfer line with buffer inventories during a period of length T, Naval Research Logistics 34 (6) (1987) 811-822.

[23] R. Mullubhatla, K. Pattipati, Discrete-time Markov reward models of automated manufacturing systems with multiple part types and random rewards, IEEE Transactions on Robotics and Automation 16 (5) (2000) 553-566.

[24] E. Muth, The Reversibility Property of Production Lines., Management Science 25 (1979) 152-158.

[25] M. Neuts, Matrix Geometric Solutions in Stochastic Models, Johns Hopkins University Press, Baltimore, 1981.

[26] J. Ou, S. B. Gershwin, The Variance of the Lead Time of a Two Machine Transfer Line with a Finite Buffer, Techical report LMP-90-028, Laboratory for Manufacturing and Productivity, MIT (1989).

[27] B. Tan, Variance of the throughput of an N-station production line with no intermediate buffers and time dependent failures, European Journal of Operational Research 101 (3) (1997) 560-576.

[28] B. Tan, An analytical formula for variance of output from a series-parallel production system with no interstation buffers and time-dependent failures, Mathematical and Computer Modelling 27 (6) (1998) 95-112.

[29] B. Tan, Effects of variability on the due-time performance of a continuous materials flow production system in series, International Journal of Production Economics 54 (1) (1998) 87-100.

[30] B. Tan, Variance of the output as a function of time: Production line dynamics, European Journal of Operational Research 117 (3) (1999) 470-484. 
[31] B. Tan, Asymptotic variance rate of the output in production lines with finite buffers, Annals of Operations Research 93 (1) (2000) 385-403.

[32] B. Tan, State-Space Modeling and Analysis of Pull-Controlled Production Systems, in: S. Gershwin, Y. Dallery, C. Papadopoulos, J. M. Smith (eds.), Analysis and Modeling of Manufacturing Systems, Operations Research Management Science, KAP, 2002.

[33] Á. Tari, M. Telek, P. Buchholz, A Unified Approach to the Moments Based Distribution Estimation - Unbounded Support, in: EPEW/WS-FM, 2005, pp. 79-93.

[34] M. Telek, Some advanced reliability modelling techniques, Phd Thesis, Hungarian Academy of Science, 1994.

[35] M. Telek, A. Heindl, Matching moments for acyclic discrete and continuous phase-type distributions of second order, International Journal of Simulation Systems, Science \& Technology 3 (3-4) (2002) 47-57.

[36] M. Telek, A. Horváth, G. Horváth, Analysis of Inhomogeneous Markov Reward Models, Linear Algebra and Its Applications 386 (2004) 383-405.

[37] M. Telek, S. Rácz, Numerical analysis of Large Markovian reward models, Performance Evaluation 36\&37 (1999) 95-114. 